\title{
AND/OR Search for Marginal MAP
}

\author{
Radu Marinescu \\ IBM Research \\ Dublin, Ireland \\ Junkyu Lee \\ Rina Dechter \\ Alexander Ihler \\ University of California, Irvine \\ Irvine, CA 92697, USA
}

RADU.MARINESCU@IE.IBM.COM

JUNKYUL@ICS.UCI.EDU

DECHTER@ICS.UCI.EDU

IHLER@ICS.UCI.EDU

\begin{abstract}
Mixed inference such as the marginal MAP query (some variables marginalized by summation and others by maximization) is key to many prediction and decision models. It is known to be extremely hard; the problem is $\mathrm{NP}^{\mathrm{PP}}$-complete while the decision problem for MAP is only NP-complete and the summation problem is \#P-complete. Consequently, approximation anytime schemes are essential. In this paper, we show that the framework of heuristic AND/OR search, which exploits conditional independence in the graphical model, coupled with variational-based mini-bucket heuristics can be extended to this task and yield powerful state-of-the-art schemes. Specifically, we explore the complementary properties of best-first search for reducing the number of conditional sums and providing time-improving upper bounds, with depth-first search for rapidly generating and improving solutions and lower bounds. We show empirically that a class of solvers that interleaves depth-first with best-first schemes emerges as the most competitive anytime scheme.
\end{abstract}

\section{Introduction}

Probabilistic graphical models provide a powerful framework for reasoning about conditional dependency structures over many variables. For such models, the Marginal MAP (MMAP) inference query is a particularly difficult yet important task, corresponding to finding the most probable configuration (or maximizing the probability) over a subset of variables, called MAP variables, after marginalizing (summing over) the remainder of the model. MMAP inference arises in many situations, especially in diagnosis and planning tasks, in which the most natural specification of the model contains many variables whose values we do not care about predicting, but which create interdependence among the variables of interest. For example, in diagnosis tasks, given observations, we seek to optimize over a subset of diagnosis variables representing potentially failing components in a system (de Kleer, Mackworth, \& Reiter, 1990; Geffner \& Bonet, 2013). Furthermore, a probabilistic conformant planning task which finds a sequence of actions that maximizes the probability of reaching a goal state without observations can be solved by MMAP inference (Lee, Marinescu, \& Dechter, 2014). More generally, the optimal policy of a finite horizon probabilistic planning task can be found by MMAP inference due to the equivalence between MMAP inference and the maximum expected utility computation (Mauá, 2016).

One reason marginal MAP queries are difficult is that the max and sum operations do not commute, forcing variable elimination to be processed along a "constrained" order that eliminates summation variables first, and often has significantly higher induced width than the graph itself 
(Dechter, 1999, 2013). In general, MMAP is $\mathrm{NP}^{\mathrm{PP}}$-complete and it can be NP-hard even on tree structured graphs (Park \& Darwiche, 2004), while the decision problem for MAP is only NP-complete (Shimony \& Charniak, 1991) and the summation problem is \#P-complete (Cooper, 1990).

The difficulty of solving marginal MAP exactly motivates anytime algorithms, which provide a confidence interval on the exact solution that is gradually improved over time. Similarly, it is important that any practical algorithm be anyspace, in the sense that it can continue to make progress once the available memory is exhausted. In this paper, we focus on the framework of heuristic search to provide these properties.

In particular, we explore several major ways in which heuristic search for marginal MAP can be made faster and more effective: using best-first techniques, exploiting AND/OR decomposition structure, and making improvements to the guiding heuristic function.

Past algorithms for solving marginal MAP have been typically based on depth-first branch and bound search, e.g., Park and Darwiche (2003), Yuan and Hansen (2009). While this approach is quick to produce an initial MAP configuration, it can be slow to improve. Evaluating or comparing any two MAP configurations involves a combinatorial sum, and when this is computationally costly, depth-first methods (which often visit a large number of such configurations) can pay a heavy price. In contrast, best-first search methods will solve far fewer summation problems to prove optimality, but will be correspondingly much slower to produce an initial solution.

The AND/OR structure provides additional improvements. AND/OR search spaces leverage conditional independence relations during search, in order to reduce the effective search space size. Although naïvely search is exponential in the number of variables, AND/OR search is exponential only in the depth of the model's pseudo-tree (Dechter \& Mateescu, 2007; Freuder \& Quinn, 1985), which may be far smaller. Moreover, if memory is utilized it can be exponential in the induced width only.

Finally, the heuristic functions used in previous work (Park \& Darwiche, 2003; Yuan \& Hansen, 2009) work by exactly solving a relaxed inference problem that follows an "unconstrained" order on the graph, which can have a smaller induced width than the exact marginal MAP task. However, this framework has two major drawbacks. First, many marginal MAP problems are not easily solved even with unconstrained orderings, limiting the methods' application in practice. We provide approximate extensions of Park and Darwiche (2003) and Yuan and Hansen (2009) that allow them to be applied more generally. More importantly, however, since the search process must follow the constrained order, these heuristics are by necessity dynamic, i.e., evaluating them at some nodes requires recomputing at least part of the variable elimination procedure, with a nontrivial computational cost. We show that a heuristic based on partition-based weighted mini-bucket (Dechter \& Rish, 2003; Liu \& Ihler, 2011; Marinescu, Dechter, \& Ihler, 2014) is much more effective for search, in part because it allows the heuristic function to be completely pre-compiled, leading to far faster per-node evaluations.

We develop AND/OR variants of both depth- and best-first search for marginal MAP. Then, motivated by our desire for good anytime behavior and the high cost of evaluating a MAP configuration, we explore two approaches that balance the benefits of depth-first and best-first behavior: weighted best-first search (Pohl, 1970; Flerova, Marinescu, \& Dechter, 2017), and two hybrid schemes that interleave best-first and depth-first operations. These algorithms demonstrate significantly better anytime performance than previous approaches.

We show that indeed heuristic search over AND/OR graphs, guided by such variational-based partition heuristic can advance exact solvers and more importantly, it results in anytime algorithms 


\begin{tabular}{|l||c|c|c|c|c|}
\hline Algorithm & Limited Memory & Strategy & Anytime & Upper Bounds & Lower Bounds \\
\hline \hline AOBB & Yes & Depth-first & Yes & No & Yes \\
AOBF & No & Best-first & No & Yes & No \\
RBFAOO & Yes & Recursive best-first & No & No & No \\
\hline BRAOBB & Yes & Depth-first & Yes & No & Yes \\
WAOBF & No & Weighted best-first & Yes & No & Yes \\
WAOBF-REP & No & Weighted best-first & Yes & No & Yes \\
WRBFAOO & Yes & Weighted best-first & Yes & No & Yes \\
\hline AAOBF & No & Best+depth-first & Yes & Yes & Yes \\
LAOBF & No & Best+depth-first & Yes & Yes & Yes \\
\hline
\end{tabular}

Table 1: New AND/OR search algorithms for marginal MAP.

for generating solutions and bounds that improve with time. Our empirical evaluations conclude that a class of solvers that interleave depth-first with best-first schemes emerges as most competitive anytime scheme.

In Table 1 we summarize the main contributions of this paper by listing the proposed AND/OR search algorithms together with their most important characteristics such as whether or not they can operate within restricted memory, search strategy, anytime behavior, as well as the ability to produce upper and lower bounds on the optimal marginal MAP value. Specifically, the first three algorithms (AOBB, AOBF and RBFAOO) denote the exact depth-first and best-first search schemes for computing optimal solutions. The subsequent four algorithms (BRAOBB, WAOBF, WAOBF-REP and WRAOBF) are the anytime schemes based on either depth-first or weighted best-first search that produce improved quality solutions (corresponding to lower bounds) in an anytime fashion. Finally, the last two algorithm (LAOBF and AAOBF) correspond to the hybrid depth+best-first search bounding schemes that compute not only anytime lower bounds but also anytime upper bounds on the optimal marginal MAP value. We will show empirically: (1) the effectiveness of the AND/OR search algorithms as exact schemes against previous unconstrained join-tree based methods exploring OR search spaces and (2) our new best+depth-first search approach produces superior quality solutions more quickly than the pure best-first or depth-first search as well as the weighted schemes.

The paper is structured as follows. In Section 2 we give notation and background on graphical models and marginal MAP. Section 3 describes some heuristic functions for marginal MAP. Then, Section 4 describes depth-first and best-first search, develops AND/OR versions for marginal MAP, and evaluates and analyzes their performance characteristics as exact solvers. Section 5 develops two families of anytime search that blend the positive qualities of depth- and best-first methods, based on using weighted best-first or interleaving best- and depth-first operations, respectively. We assess the performance of these two frameworks on a number of benchmark problem classes. Finally, we describe some connections to related work in Section 6, and discuss our conclusions in Section 7. The paper is a significantly expanded and unified presentation of a line of work by the authors, including (Marinescu et al., 2014; Marinescu, Dechter, \& Ihler, 2015; Lee, Marinescu, Dechter, \& Ihler, 2016; Marinescu, Lee, Dechter, \& Ihler, 2017). 


\section{Background}

A graphical model is a tuple $\mathcal{M}=\langle\mathbf{X}, \mathbf{D}, \mathbf{F}\rangle$, where $\mathbf{X}=\left\{X_{i}: i \in V\right\}$ is a set of variables indexed by set $V$ and $\mathbf{D}=\left\{D_{i}: i \in V\right\}$ is the set of their finite domains of values. $\mathbf{F}=\left\{\psi_{\alpha}: \alpha \in F\right\}$ is a set of discrete positive real-valued local functions defined on subsets of variables, where we use $\alpha \subseteq V$ and $\mathbf{X}_{\alpha} \subseteq \mathbf{X}$ to indicate the scope of function $\psi_{\alpha}$, ie, $\mathbf{X}_{\alpha}=\operatorname{var}\left(\psi_{\alpha}\right)=\left\{X_{i}: i \in \alpha\right\}$. The function scopes imply a primal graph whose vertices are the variables and which includes an edge connecting any two variables that appear in the scope of the same function. The graphical model $\mathcal{M}$ defines a factorized probability distribution on $\mathbf{X}$, namely

$$
P(\mathbf{X})=\frac{1}{Z} \prod_{\alpha \in F} \psi_{\alpha}
$$

The partition function, $Z$, normalizes the probability to sum to one.

Marginal MAP problems distinguish maximization variables (called MAP variables) and summation variables (the others). Let $\mathbf{X}_{S}$ be the subset of summation variables in $\mathbf{X}$ and $\mathbf{X}_{M}=\mathbf{X} \backslash \mathbf{X}_{S}$ be the complement of $\mathbf{X}_{S}$. The Marginal MAP problem is to find the assignment $x_{M}^{*}$ to variables $\mathbf{X}_{M}$ that maximizes the value of the marginal distribution after summing out variables $\mathbf{X}_{S}$ :

$$
x_{M}^{*}=\arg \max _{\mathbf{X}_{M}} \sum_{\mathbf{X}_{S}} \prod_{\alpha \in F} \psi_{\alpha}\left(x_{\alpha}\right)
$$

Example 1. Figure 1(a) depicts the primal graph of an undirected graphical model (called also a Markov network) representing a distribution over 8 variables, $\mathbf{X}=A, \ldots, H$ with 8 functions defined by the arcs (each pair is a scope of one function). The highlighted variables $\{A, B, C, D\}$ denotes the MAP variables, in a possible marginal MAP task.

\subsection{Bucket Elimination}

An exact solution to (1) can be obtained by using the bucket elimination (BE) algorithm, which eliminates (sums or maximizes over) the variables in sequence (Dechter, 1999). Given an ordering of the variables in which all the max variables come before the sum variables, BE partitions the functions into buckets, each associated with a single variable. A function is placed in the bucket of its argument that appears latest in the ordering. BE processes each bucket, from last to first, by multiplying all functions in the current bucket and eliminating the bucket's variable (by summation for sum variables and by maximization for MAP variables), resulting in a new function which is then placed in an earlier bucket, depending on its scope. Since the size of this function is exponential in its number of arguments, the complexity of $\mathrm{BE}$ is time and space exponential in the induced width of the primal graph along the elimination order (Dechter, 1999).

Because the order in a marginal MAP task is constrained to place all max variables before all sum variables, we refer to it as a constrained elimination order, and to its induced width as the constrained induced width, denoted $w_{c}^{*}$. The constrained induced width is always higher than the induced width of the best unconstrained order, sometimes significantly so. Marginal MAP is harder than pure optimization (MAP) tasks because evaluating the cost of the MAP assignment requires solving a summation over the sum variables, and harder than pure summation because it may force an undesirable (constrained) elimination order. For example, while summation is efficient in tree-structured graphs (induced width 1), it is easy to specify MAP variables such that the resulting 


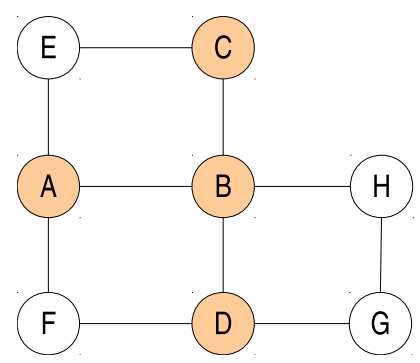

(a) Primal graph

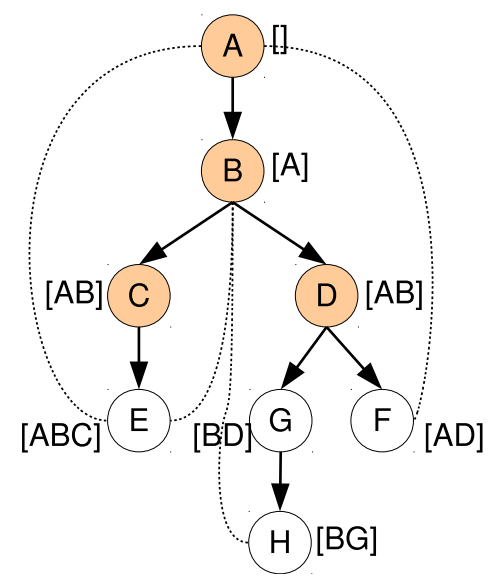

(b) Pseudo-tree

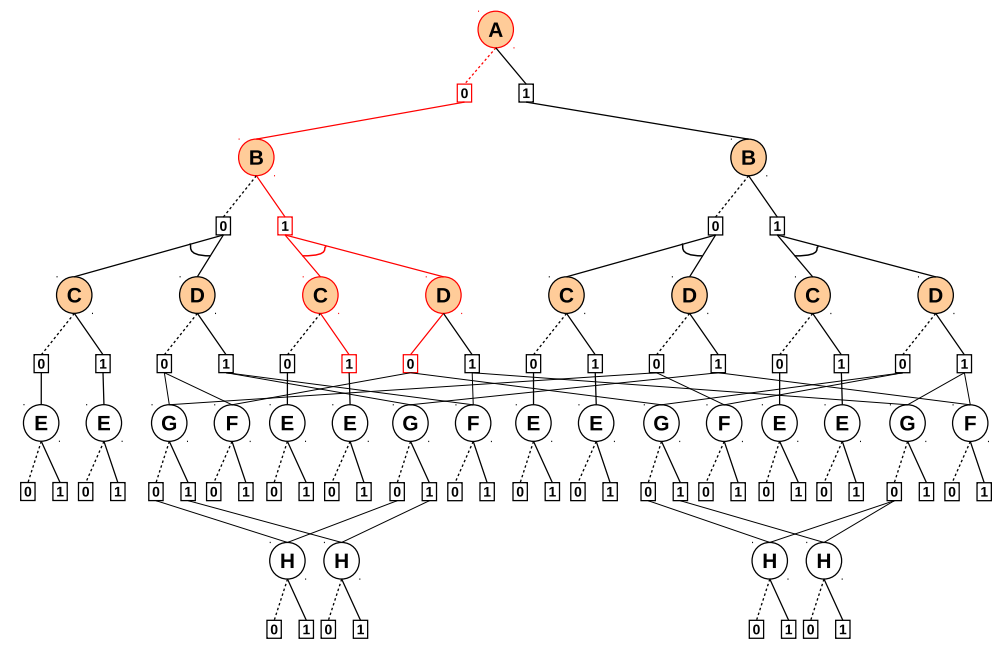

(c) AND/OR search graph

Figure 1: Example (a) primal graph of a graphical model over eight variables; (b) a pseudo-tree corresponding to the ordering $d=(A, B, C, D, E, G, F, H)$; (c) the resulting AND/OR search graph. An example solution tree is indicated in red (see Section 4.2).

marginal MAP problem is NP-hard. This effect means that many marginal MAP problems are impractical to solve exactly through bucket elimination, necessitating approximations and search methods.

\subsection{AND/OR Search Spaces}

AND/OR search is guided by a spanning pseudo-tree of the graphical model's primal graph (in which any arc of the model not in the tree is a back-arc in the pseudo-tree) and the search space is exponential in the depth of the pseudo-tree (rather than in the number of variables). Therefore, any of the inference tasks over a graphical model can be computed by traversing the AND/OR search tree (Dechter \& Mateescu, 2007) even with linear memory. Moreover, when memory can be used the 
AND/OR search space can be compacted into a graph whose size is exponential in the induced width only.

DEFINITION 1 (pseudo-tree). A pseudo-tree of an undirected graph $G=(V, E)$ is a directed rooted tree $\mathcal{T}=\left(V, E^{\prime}\right)$ such that every arc of $G$ not included in $E^{\prime}$ is a back-arc in $\mathcal{T}$, namely it connects a node in $\mathcal{T}$ to one of its ancestors. The arcs in $E^{\prime}$ may not all be included in $E$.

Figure 1(b) shows a pseudo-tree of depth 5 of the graphical model in Figure 1(a) that could be generated by a depth-first traversal of the graph in Figure 1(a) (for more details on how to construct pseudo-trees, see Dechter and Mateescu (2007), Marinescu and Dechter (2009a)). Given a graphical model $\mathcal{M}=\langle\mathbf{X}, \mathbf{D}, \mathbf{F}\rangle$ with primal graph $G$ and pseudo-tree $\mathcal{T}$ of $G$, the $A N D / O R$ search tree $S_{\mathcal{T}}$ based on $\mathcal{T}$ has alternating levels of OR nodes corresponding to the variables and AND nodes corresponding to the values of the OR parent's variable, with edges weighted according to $\mathbf{F}$. The AND/OR search space can be compacted further by merging nodes that root identical subtrees. One way to identify such nodes is by using the notion of context (Dechter \& Mateescu, 2007).

DEFINITION 2 (parents, context). Given a graphical model $\mathcal{M}$ with primal graph $G$ and pseudo-tree $\mathcal{T}$, the parents of variable $X_{i}$, denoted by $p a_{i}$ or $p a_{X_{i}}$, are the ancestors of $X_{i}$ in $\mathcal{T}$ that have connections (in $G$ ) to $X_{i}$ or to descendants of $X_{i}$ in $\mathcal{T}$. Let $n$ be an OR node labeled by variable $X_{i}$ in the AND/OR search tree $S_{\mathcal{T}}$. A variable assignment to $p a_{i}$ defines the context of node $n$.

It was shown that any two OR nodes $n_{1}$ and $n_{2}$ with identical contexts root identical subproblems in $S_{\mathcal{T}}$ and therefore can be merged (Dechter \& Mateescu, 2007). Merging all nodes that have identical contexts yields the context-minimal AND/OR graph shown in Figure 1(c) (the parents sets of the variables are shown next to each node of the pseudo-tree in Figure 1(b)). The size of this search space can be bounded exponentially by the treewidth $w^{*}$ (or, the induced width). Searching the AND/OR context-minimal graph can be bounded to be time and space exponential in the treewidth $w^{*}$, the same complexity bound obeyed by message-passing schemes such as bucket elimination (Dechter, 1999). Yet, search schemes can work with more flexible representations which is not necessarily table-based, and invite methods that can trade memory for time and lead smoothly into anytime schemes.

\section{Improved Partitioning Based Upper Bounds for Marginal MAP}

Informed search algorithms rely heavily on a heuristic function to guide the search process, and bound the quality of potential solutions. In practice, these heuristics are usually constructed by approximating the exact bucket elimination process described in Section 2. In this section, we describe two common frameworks for heuristic construction: mini-bucket (Dechter \& Rish, 2003) and reordered join-tree (Park \& Darwiche, 2003) heuristics. Both frameworks have drawbacks: in particular, while the reordered heuristic has been shown to outperform mini-bucket, its computational complexity is not easily controlled, and by construction it can require significant recomputation at some nodes of the search. We fix the former issue using a heuristic called mini-cluster-tree, which further approximates the reordered join-tree in a computationally bounded way. In order to develop a more efficient heuristic, we revisit mini-bucket and augment it with several recent developments to improve its quality on pure summation (Liu \& Ihler, 2011) and pure MAP (Ihler, Flerova, Dechter, \& Otten, 2012), giving a weighted mini-bucket heuristic for marginal MAP that can be used in AND/OR search. This provides a stronger heuristic than standard mini-bucket, while remaining highly efficient to evaluate at each node. 


\subsection{Mini-Bucket Elimination}

Mini-Bucket Elimination (MBE) is a classic relaxation of the exact variable elimination problem in (1) by approximating each elimination operator (max or sum) to enable the user to control a bound on the space and time complexity. Following the exact bucket elimination procedure, each bucket is further partitioned into smaller subsets, called mini-buckets, each containing at most $i$ distinct variables (where $i$ is a user-selected parameter called the $i$-bound). The mini-buckets are processed independently, resulting in messages over fewer variables and thus require less time and memory. MBE processes the sum buckets and max buckets differently. Max mini-buckets (of variables in $\mathbf{X}_{M}$ ) are eliminated by maximization, while for variables in $\mathbf{X}_{S}$, one (arbitrarily selected) mini-bucket is eliminated by summation, while the rest of the mini-buckets are eliminated by maximization. The complexity of the algorithm is time and space exponential only in its $i$-bound, which can be selected to enforce available computational resources. When $i$ is sufficiently large (i.e., $i>w_{c}^{*}$ ), MBE coincides with full bucket elimination, yielding the exact answer.

By design, these mini-bucket messages upper bound the exact elimination process, giving an upper bound on the optimal marginal MAP value. Moreover, given any partial configuration that respects this ordering (i.e., consists of values for the last $k$ eliminated variables), we can evaluate an upper bound on the conditional problem from the messages computed during the approximate elimination. These bounds can be used as a search heuristic, and are very efficient to evaluate, requiring at most a few table lookup operations per search node. $\operatorname{MBE}(i)$ has regularly been used as an effective heuristic for depth-first branch and bound search on MAP problems (Marinescu \& Dechter, 2009a, 2009b).

\subsection{Unconstrained-order, Join-tree Based Upper Bounds}

An alternative approach for upper bounding marginal MAP was proposed by Park and Darwiche (2003). In this method, a join-tree is constructed along an unconstrained elimination order that interleaves the MAP and the sum variables. Since reversing the order of the sum and max elimination in (1) gives an upper bound on its value, a standard two-pass evaluation of the join-tree for this reordered problem is guaranteed to generate an upper bound on the optimal marginal MAP value. The complexity of the evaluation is exponential in the unconstrained induced width of the underlying primal graph, which can be less than the constrained induced width. When this computational cost is not too high, this method was shown to be more effective than MBE (Park \& Darwiche, 2003).

Unfortunately, there are two significant drawbacks to this approach. The first is that the computational requirements are not completely under user control. In many models, even the unconstrained induced width remains prohibitively high, which limits the method's practical application. In order to use these techniques in general models, we may need to further approximate them; in the sequel, we develop a mini-cluster tree elimination heuristic to overcome this issue.

The second drawback of unconstrained order methods is that they are by definition dynamic, meaning that, at some search nodes, they may require some significantly non-trivial computation to evaluate the heuristic bound. Yuan and Hansen (2009) improve on (Park \& Darwiche, 2003), reducing the number of such re-evaluations by carefully caching and reusing messages, but still require inference re-computation at some nodes. Fundamentally, this is a consequence of the fact that the search must proceed over the max variables; whenever a re-ordered max variable is conditioned on in the search, the join tree messages no longer provide a usable heuristic and must be re-computed. 
For this reason, we also propose an improved form of (constrained-order) mini-bucket heuristic for marginal MAP.

\subsection{Mini-Cluster-Tree Elimination}

To control the computational costs of the unconstrained order based heuristic, we apply an approximation with bounded complexity, related to mini-bucket but with computations organized more similarly to join-tree inference, called Mini-Cluster-Tree Elimination (MCTE) (Dechter, Kask, \& Larrosa, 2001). In MCTE, we pass messages along the structure of the join-tree, except that when computing a message, rather than combining all the functions in the cluster, we first partition it into mini-clusters, such that each mini-cluster has a bounded number of variables (the $i$-bound). Each mini-cluster is then processed independently to compute a set of outgoing messages. Like MBE, this procedure produces an upper bound on the results of exact inference, and increasing $i$ typically provides tighter bounds, but at higher computational cost. In spirit, both MBE and MCTE are quite similar and both methods could be applied to either constrained or unconstrained order eliminations, but in this paper for consistency we always use MBE-based relaxations on constrained orderings, and MCTE relaxations on unconstrained orderings.

The dynamically evaluated nature of reordered (unconstrained order) heuristics, however, is more fundamental to the approach. While dynamic heuristics are often tighter than statically compiled ones, and can enable additional, useful techniques such as dynamic search orders, the efficiency of evaluating a static heuristic allows it to visit more nodes in a given time. For this reason, we explore an improved static heuristic based on weighted mini-bucket elimination, which we show experimentally leads to a more effective search process.

\subsection{Weighted Mini-Bucket Elimination and Cost-shifting}

Weighted Mini-Bucket (WMB) (Liu \& Ihler, 2011) is a recent enhancement of the mini-bucket scheme for likelihood (summation) tasks. It replaces the naïve mini-bucket bound with Hölder's inequality. For a given variable $X_{k}$, the mini-buckets $Q_{k r}$ associated with $X_{k}$ are each assigned a non-negative weight $w_{k r} \geq 0$, such that $\sum_{r} w_{k r}=1$. Then, each mini-bucket $r$ is processed using a weighted or power sum, $\left(\sum_{X_{k}} f(X)^{1 / w_{k r}}\right)^{w_{k r}}$. It is useful to note that $w_{k r}$ can be interpreted as a "temperature"; if $w_{k r}=1$, the power sum corresponds to a standard summation, while if $w_{k r} \rightarrow 0$, it instead corresponds to a maximization over $X_{k}$. Thus, standard mini-bucket for summation queries corresponds to choosing one mini-bucket $r$ with $w_{k r}=1$, and the rest with weight zero.

Weighted mini-bucket is closely related to variational bounds on the likelihood, such as conditional entropy decompositions (Globerson \& Jaakkola, 2007) and tree-reweighted belief propagation (TRBP) (Wainwright, Jaakkola, \& Willsky, 2005). The single-pass algorithm of Liu and Ihler (2011) mirrors standard mini-bucket, except that within each bucket a cost-shifting (or reparameterization) operator is performed, which matches the marginal beliefs (or "moments") across mini-buckets to improve the bound.

The temperature viewpoint of the weights enables a similar procedure for marginal MAP. In particular, for $X_{k} \in \mathbf{X}_{S}$, we enforce $\sum_{r} w_{k r}=1$, while for $X_{k} \in \mathbf{X}_{M}$, we take $\sum_{r} w_{k r}=0$ (so that $w_{k r}=0$ for all $r$ ). The resulting algorithm, presented in Algorithm 1 and called Weighted Mini-Bucket with Moment Matching (WMB-MM(i)), treats MAP and sum variables differently: for sum variables it is like Liu and Ihler (2011), while taking the zero-temperature limit for MAP variables we obtain the max-marginal matching operations described for pure MAP problems in 


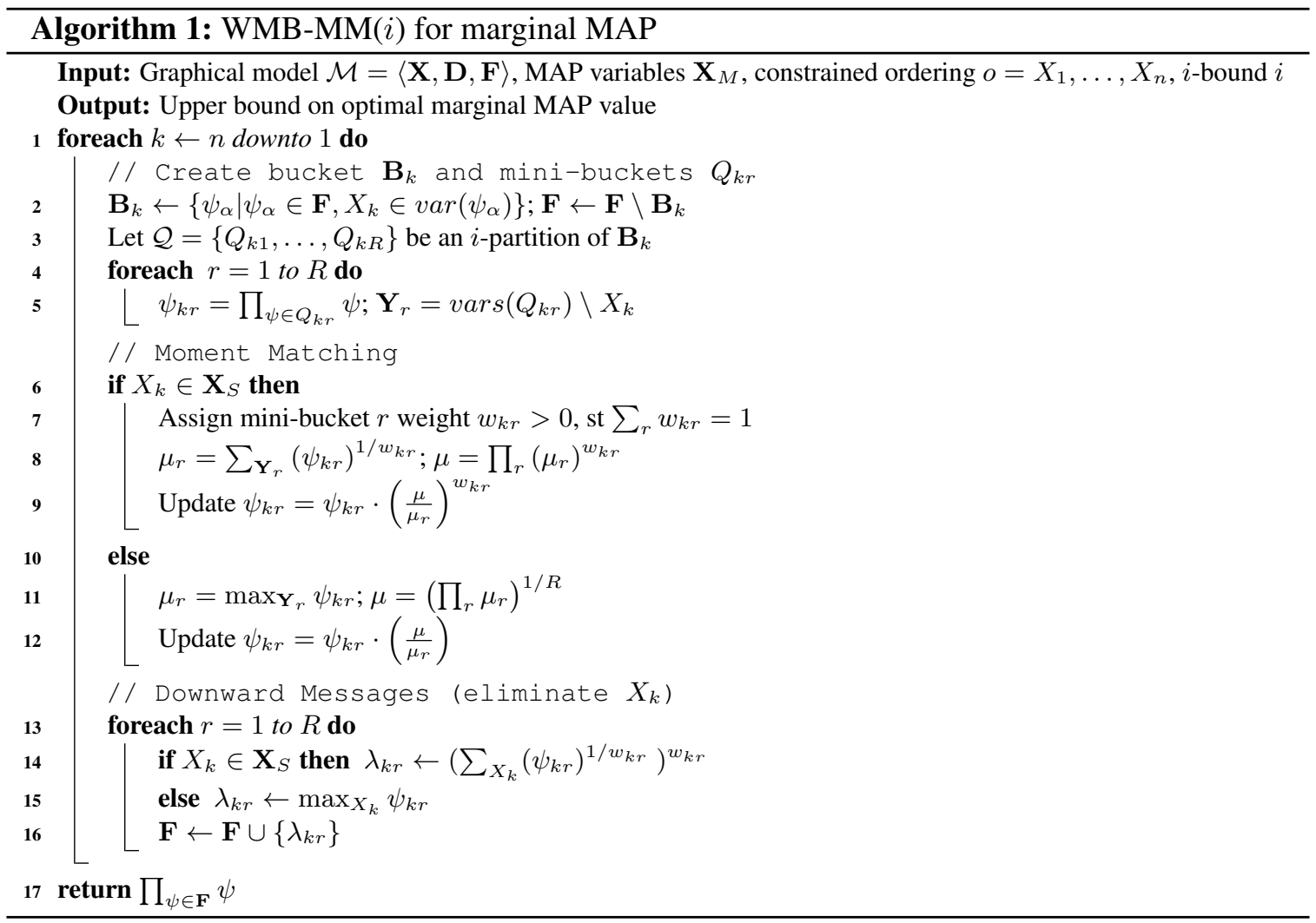

(Ihler et al., 2012). This mirrors the result of (Weiss, Yanover, \& Meltzer, 2007), that the linear programming relaxation for MAP corresponds to a zero-temperature limit of TRBP.

The bounds provided by the single-pass WMB-MM $(i)$ algorithm can be further tightened, if desired, by additional message passing. One example is the Weighted Mini-Buckets with Join Graph propagation algorithm, WMB-JG $(i)$, which alternates between downward passes similar to Algorithm 1 and upward passes, which compute messages used to "focus" the cost-shifting in the next downward pass; see (Marinescu et al., 2014) for additional details. Another, more distributed update scheme generalizing dual decomposition methods is given in (Ping, Liu, \& Ihler, 2015).

Although iterative methods can produce tighter upper bounds compared to the single-pass WMB$\operatorname{MM}(i)$, when used in practice as a heuristics generator for search, the computational overhead associated with iterative schemes such as WMB-JG often outweighs the improved accuracy of its bounds (Marinescu et al., 2014). Therefore, in the sequel we use WMB-MM( $i$ ) as the heuristic to guide our various AND/OR search algorithms for marginal MAP.

\section{AND/OR Search Algorithms for Marginal MAP}

In this section, we introduce our search algorithms for marginal MAP. As noted, these algorithms explore the AND/OR search space for marginal MAP and are guided by effective weighted minibucket heuristics improved with cost shifting schemes. Specifically, we develop depth-first AND/OR branch and bound and best-first AND/OR search algorithms that explore the context minimal AND/OR search graph. Since memory can be a bottleneck, especially for best-first search, we also 
develop a recursive best-first AND/OR search algorithm that can operate efficiently within bounded memory. We first review search methods for marginal MAP which precede our work.

\subsection{Early Search Methods}

Marginal MAP can be solved by traversing either depth-first or best-first an OR search space defined by the MAP variables. The search can be further enhanced with a specialized heuristic that upper bounds the value of each partial MAP assignment, thus allowing the use of standard pruning methods such as branch and bound. Up until recently, the best performing algorithm for marginal MAP was the depth-first branch and bound developed by Park and Darwiche (2003) which uses the unconstrained join-tree upper bound for guidance. During search, the join-tree is fully re-evaluated at each node in order to compute upper bounds for all uninstantiated MAP variables simultaneously, which allows the use of dynamic variable ordering. Although this approach provides effective bounds, the computational overhead at each node in the search space can be quite significant. Subsequently, Yuan and Hansen (2009) proposed an incremental evaluation of the join-tree bounds which significantly reduces the computational overhead. However, this modification also requires the search algorithm to follow a static variable ordering. In practice, Yuan and Hansen's method proved to be cost effective, considerably outperforming Park and Darwiche (2003). However, both methods require that the induced width of the unconstrained join tree to be small enough to be feasible.

\subsubsection{DEPTH-FIRST BRANCH AND BOUND}

We next describe two algorithms that generalize the Park and Darwiche (2003) and Yuan and Hansen (2009) schemes for models with high unconstrained induced width. In particular, we use MCTE $(i)$ (Dechter et al., 2001) to approximate the exact, unconstrained join-tree inference to accommodate a maximum clique size defined by the $i$-bound $i$. The resulting branch and bound with $\operatorname{MCTE}(i)$ unconstrained heuristics, abbreviated BBBT ${ }^{1}$, is given in Algorithm 2

The algorithm searches the simple OR tree of all partial MAP variable assignments. At each step, BBBT uses $\operatorname{MCTE}(i)$ over an unconstrained ordering to compute an upper bound $U(\bar{x})$ on the optimal extension of the current partial MAP assignment $\bar{x}$ (lines 5-8). If $U(\bar{x}) \leq L$, the current best solution cost, then the current assignment $\bar{x}$ cannot lead to a better solution and the algorithm can backtrack (line 9). Otherwise, BBBT expands the current assignment by selecting the next MAP variable in a static or dynamic variable ordering (line 4) and recursively solves a set of subproblems, one for each un-pruned domain value. Notice that when $\bar{x}$ is a complete assignment, BBBT calculates its marginal MAP value by solving a summation task over $\left.\mathcal{M}\right|_{\bar{x}}$, the subproblem defined by the sum variables conditioned on $\bar{x}$ (line 2). Given sufficient resources (high enough $i$-bound), this can be done by variable elimination or by depth-first AND/OR search with caching (Dechter \& Mateescu, 2007) (see also Section 4.3). If a better new assignment is found then the lower bound $L$ is updated (line 10).

If $\operatorname{MCTE}(i)$ is fully re-evaluated at each iteration, it produces upper bounds for all uninstantiated MAP variables simultaneously. In this case, BBBT can accommodate dynamic variable orderings and can thus be viewed as a generalization of Park and Darwiche (2003). Alternatively, $\operatorname{MCTE}(i)$ can be done in an incremental manner to provide a generalization of Yuan and Hansen (2009); in this case BBBT requires a static variable ordering.

1. For consistency with prior work, we use the name used in Marinescu et al. (2003), (branch and bound with Bucket-Tree heuristic) to denote the same algorithm applied to pure MAP queries. 


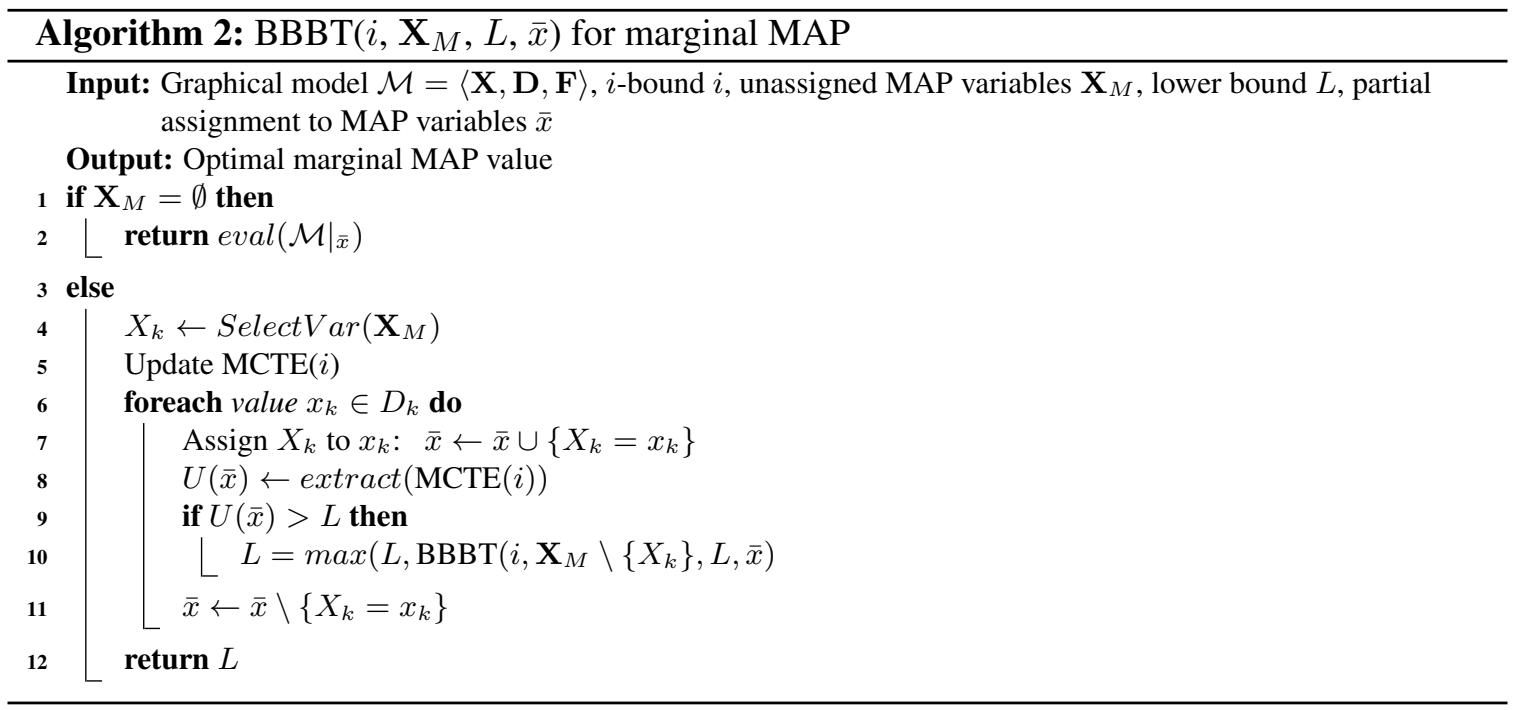

\subsubsection{BeST-First SEARCH}

Best-first search schemes (e.g., $\mathrm{A}^{*}$ ) are known to be superior to other search schemes that traverse the same search space given the same heuristic information (Dechter \& Pearl, 1985). Algorithm A* expands only nodes satisfying $f(n) \geq C^{*}$ (for maximization) where $C^{*}$ is the cost of the optimal solution and where $f(n)=g(n)+h(n)$ (for sum cost paths), and $h(n)$ is an upper bound of the best cost extension to a solution (Nilsson, 1980; Pearl, 1988). Any complete search algorithm, and in particular, depth-first branch and bound (DFBnB), must explore all the nodes expanded by $\mathrm{A}^{*}$, assuming the same tie breaking rule, and, in addition may also explore some nodes for which $f(n)<C^{*}$. Therefore, the expected benefit of $\mathrm{A}^{*}$ over any other search algorithm, $B$, exploring the same search space, depends on the number of extra nodes explored by $B$.

To understand A*'s performance for marginal MAP inference, we define an OR search space whose nodes are partial assignments to a subset of the MAP variables along a fixed order $X_{1}, \ldots, X_{m}$. To capture the summation operation applied over $\mathbf{X}_{S}$, we introduce a final solution/goal node denoted $s$, which extends any full MAP assignment. A solution path in this search space can be denoted by $\left(x_{1}, \ldots, x_{m}, s\right)$. Formally,

DEFINITION 3 (OR MMAP search space). Let $\mathbf{X}_{M}$ be the MAP variables of a graphical model $\mathcal{M}=\langle\mathbf{X}, \mathbf{D}, \mathbf{F}\rangle$ and $\mathbf{X}_{S}=\mathbf{X} \backslash \mathbf{X}_{M}$. The search space for MMAP has nodes which are partial assignments over $\mathbf{X}_{M}$, denoted $\bar{x}_{1 . . j}=\left(x_{1}, \ldots, x_{j}\right)$. All full MAP assignments have the same child node, s. All the arc-weights of internal nodes are extracted from the functions $\mathbf{F}$. Specifically, the weight of the arc $\left(\bar{x}_{1 . . l-1} \rightarrow \bar{x}_{1 . . l}\right)$ is the product of all the functions $F\left(X_{l}\right) \subseteq F$ whose scope includes $X_{l}$ and is completely instantiated by the partial assignment $\bar{x}_{1 . . l}$, namely $w\left(\bar{x}_{1 . . l-1} \rightarrow\right.$ $\left.\bar{x}_{1 . . l}\right)=\prod_{\alpha \in F\left(X_{l}\right)} \psi_{\alpha}\left(\mathbf{x}_{\alpha} \mid \bar{x}_{1 . . l}\right)$. The arc-weight connecting a full MAP assignment $\mathbf{x}_{M}$ node to $s$ is defined by:

$$
w\left(\mathbf{x}_{M} \rightarrow s\right)=\sum_{\mathbf{X}_{S}} \prod_{\alpha \in F\left(\mathbf{X}_{S}\right)} \psi_{\alpha}\left(\mathbf{x}_{\alpha} \mid \mathbf{x}_{M}\right)
$$

where $F\left(\mathbf{X}_{S}\right) \subseteq F$ is the subset of function scopes that include summation variables in $\mathbf{X}_{S}$.

It is easy to show that, 
Proposition 1. Given a graphical model $\mathcal{M}=\langle\mathbf{X}, \mathbf{D}, \mathbf{F}\rangle$ and MAP variables $\mathbf{X}_{M}$, any search algorithm finding an optimal solution path over its MMAP search space, finds an optimal solution to its MMAP task.

Proof. Let $\pi=\left(x_{1}, \ldots, x_{m}, s\right)$ be a solution path in the MMAP search space. By definition 3, the cost of $\pi$ obeys $c(\pi)=\prod_{l=1}^{m} \prod_{\alpha \in F\left(X_{l}\right)} \psi\left(\mathbf{x}_{\alpha} \mid \bar{x}_{1 . . l}\right) \cdot \sum_{\mathbf{x}_{S}} \prod_{\alpha \in F\left(\mathbf{X}_{S}\right)} \psi\left(\mathbf{x}_{\alpha} \mid \bar{x}_{1 . . m}\right)$. Let $F\left(\mathbf{X}_{M}\right) \subseteq$ $F$ be the subset of function scopes that are defined on MAP variables only. It follows easily that $c(\pi)=\prod_{\alpha \in F\left(\mathbf{X}_{M}\right)} \psi_{\alpha}\left(\mathbf{x}_{\alpha} \mid \bar{x}_{1 . . m}\right) \cdot \sum_{\mathbf{X}_{S}} \prod_{\alpha \in F\left(\mathbf{X}_{S}\right)} \psi\left(\mathbf{x}_{\alpha} \mid \bar{x}_{1 . . m}\right)$ and since $F=F\left(\mathbf{X}_{M}\right) \cup F\left(\mathbf{X}_{S}\right)$ we obtain $c(\pi)=\sum \mathbf{x}_{S} \prod_{\alpha \in F} \psi\left(\mathbf{x}_{\alpha} \mid \bar{x}_{1 . . m}\right)$. The optimal solution path $\pi^{*}$ is $\pi^{*}=\operatorname{argmax}_{\pi} c(\pi)=$ $\operatorname{argmax}_{\bar{x}_{1 . . m}} \sum \mathbf{X}_{S} \prod_{\alpha \in F} \psi\left(\mathbf{x}_{\alpha} \mid \bar{x}_{1 . . m}\right)$ which is the optimal solution to the MMAP task.

The most costly operation when traversing even a single solution path in the MMAP search space is when the algorithm expands a full MAP assignment $\bar{x}_{1 . . m}$, called a frontier MAP node. This is because it needs to evaluate the last arc-weight, which is the cost of this MAP assignment, by a summation problem.

Thus, a search algorithm that minimizes such expansions can be significantly more effective. In fact, we argue that $\mathrm{A}^{*}$ 's potential for marginal MAP is likely to be more profound than in regular optimization (e.g., for pure MAP) because it can save not only on the number of regular nodes expanded, but (much more significantly) on the number of expansions of MAP frontier nodes, and therefore the number of summation sub-tasks.

THEOREM 1. Let $A^{*}$ be a search algorithm exploring the OR MMAP search space and let $B$ be any optimal search algorithm that explores the same search space as $A^{*}$. Given the same heuristic function and the same tie breaking rule, we have that:

(a) any node expanded by $A^{*}$ will be expanded by $B$.

(b) any MAP frontier node expanded by $A *$ must be expanded by $B$.

Proof. When A* terminates with a path $\left(x_{1}, \ldots, x_{m}, s\right)$ it has found the optimal MAP solution.

Denote by $R$ the set of full MAP assignments, of the form $\left(x_{1}, \ldots, x_{m}\right)$ in OPEN at the moment A* terminates (namely, those nodes which were evaluated but not expanded). Denote by $S$ the full solutions in OPEN, having the form $\left(x_{1}, \ldots, x_{m}, s\right)$. Any node in $S$ represents an evaluation of the summation sub-task. From the known optimality of A* (Nilsson, 1980), it follows that all the nodes in $S$ must also be evaluated by $B$, and in particular, by $\mathrm{DFBnB}$, and all have $f \geq C^{*}$ where $C^{*}$ is the cost of an optimal MAP assignment.

We argue that a DFBnB scheme is likely to expand extra MAP frontier nodes beyond $S$, some of which have $f<C^{*}$. While extra node expansions occur in any regular path-finding DFBnB search, they are normally easy to control. This is because DFBnB is often supplied with a near-optimal solution (e.g., using local search) which closes the gap between its best current solution $L$ and $C^{*}$, and thus prunes the search space almost as effectively as $\mathrm{A}^{*}$ (since $f(n)<L$ is close to $f(n)<C^{*}$ ). However, finding an initial good solution (and thus a good lower bound) is far harder in the context of marginal MAP as there is no simple and effective local search scheme. For these reasons, the superiority of $\mathrm{A}^{*}$ over DFBnB is potentially more significant for MMAP. 


\subsection{AND/OR Search Spaces for Marginal MAP}

The AND/OR search space for marginal MAP is defined relative to a valid pseudo-tree, in which the MAP variables are grouped at the top of the pseudo-tree so that they form a start pseudo tree, or a subtree of pseudo-tree $\mathcal{T}$ that has the same root as $\mathcal{T}$.

Given a context-minimal AND/OR graph $C_{\mathcal{T}}$ relative to a valid pseudo-tree $\mathcal{T}$, a solution tree $\hat{x}$ of $C_{\mathcal{T}}$ is a subtree defined over the MAP variables only such that: (1) it contains the root of $C_{\mathcal{T}}$; (2) if an internal OR node $n \in C_{\mathcal{T}}$ is in $\hat{x}$, then $n$ is labeled by a MAP variable and exactly one of its children is in $\hat{x}$; (3) if an internal AND node $n \in C_{\mathcal{T}}$ is in $\hat{x}$ then all its OR children labeled by MAP variables are in $\hat{x}$.

Each node $n$ in $C_{\mathcal{T}}$ can be associated with a value $v(n)$; for MAP variables, $v(n)$ captures the optimal marginal MAP value of the conditioned subproblem rooted at $n$, while for sum variables it is the conditional likelihood (sum) of the subproblem. Clearly, $v(n)$ can be computed recursively based on the values of $n$ 's successors: OR nodes by maximization or summation (for MAP or sum variables, respectively), and AND nodes by multiplication.

It is important to notice that, by definition, the AND/OR search space for marginal MAP spans both the MAP as well as the summation variables. Therefore, the AND/OR search algorithms presented in this section and the next can exploit decomposition in the MAP part of the problem as well as in the corresponding conditioned summation part.

Example 2. Consider again the context-minimal AND/OR search graph from Figure 1(c) relative to the valid pseudo-tree from Figure 1(b). The parents sets of the variables (which define the node contexts in the search space) are shown next to the pseudo-tree nodes. It is easy to see that the MAP variables form a start pseudo-tree. A solution tree corresponding to the MAP assignment $(A=0, B=1, C=1, D=0)$ is indicated in red.

\subsection{AOBB: Depth-First AND/OR Branch and Bound}

We are now ready to describe our depth-first AND/OR branch and bound (AOBB) algorithm for marginal MAP. The idea is to traverse the AND/OR search space in a depth-first manner and update the node values recursively based on the values of their successors. In order to guide the search more effectively, each node $n$ in the search space that is labeled by a MAP variable is associated with a heuristic estimate $h(n)$ which provides an upper bound on $v(n)$. These estimates are then used to prune unpromising regions of the search space. AOBB is presented in Algorithm 3.

We use the notation that $T_{s}^{\prime}$ is the current partial solution subtree, $s$ is the root node of the search space and the table Cache, indexed by node contexts, holds the partial search results. The fringe of the search is maintained by a stack called $O P E N$. The algorithm assumes that variables are selected in a static order respecting a valid pseudo-tree $\mathcal{T}$. A heuristic $f\left(T_{m}^{\prime}\right)$ calculates an upper bound on the optimal marginal MAP extension of $T_{m}^{\prime}$, where $T_{m}^{\prime}$ is a partial solution subtree rooted at node $m$ ( $T_{m}^{\prime}$ is also a subtree of $T_{s}^{\prime}$ and $m \in T_{s}^{\prime}$ ). Each OR node $n$ is associated with a flag n.optimal which, if true, indicates that the subproblem rooted by $n$ was solved optimally.

AOBB interleaves a top-down forward expansion of the current partial solution tree with a bottom-up cost revision step that updates the node values. It selects the tip node $n$ on top of the search stack and, if $n$ is labeled by a MAP variable, the algorithm attempts to prune the search space below it (lines 4-10). Specifically, it computes the heuristic evaluation function for every partial solution subtree rooted at the OR ancestors of $n$ along the path to the root. The search below $n$ is terminated if, for some ancestor $m, f\left(T_{m}^{\prime}\right) \leq v(m)$, where $v(m)$ is the current lower bound on the 


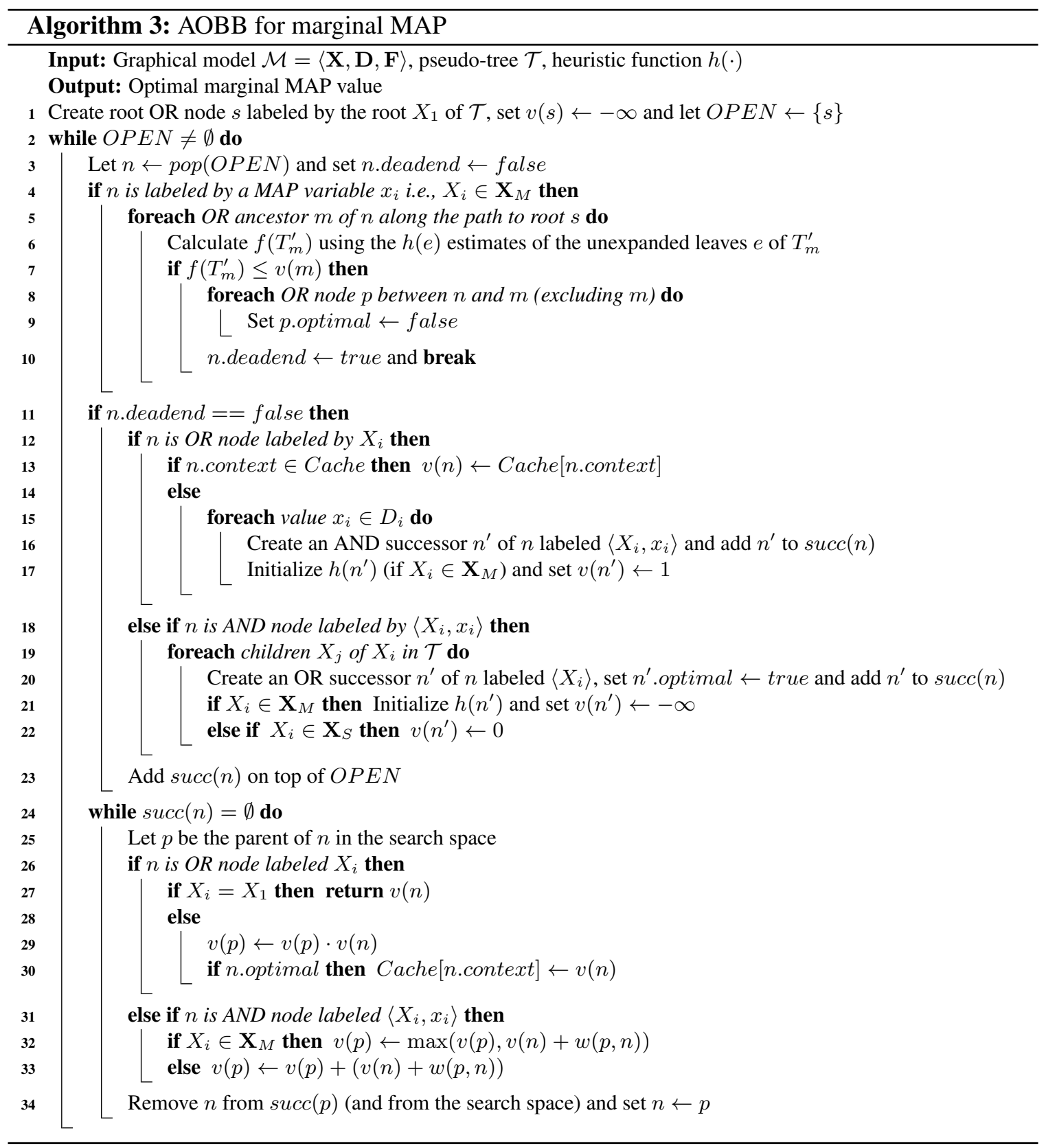

optimal value below $m$. The algorithm also labels as not optimal all of $n$ 's OR ancestors between $n$ and $m$ (excluding $m$ ) indicating that it is not safe to cache the values of these nodes. It is easy to see that AOBB ensures only provably optimal OR node values are cached and reused during search.

If node $n$ was not pruned then the algorithm expands it by generating its successors. If $n$ is an OR node, labeled by $X_{i}$, then its successors are AND nodes represented by the values $x_{i}$ in variable $X_{i}$ 's domain (lines 12-17). Notice that at this stage the algorithm checks the cache table and if the same context n.context was encountered before it is retrieved from cache which will trigger the 
propagation step. If $n$ is an AND node labeled $\left\langle X_{i}, x_{i}\right\rangle$, then its successors are OR nodes labeled by the child variables of $x_{i}$ in the pseudo-tree $\mathcal{T}$ (lines 18-22).

The node values are updated by a bottom-up propagation step (lines 24-34) which is triggered when a node $n$ has an empty set of successors. Note that as each successor is evaluated, it is removed from the set of successors in line 34. This means that all of $n$ 's children have been evaluated and their final node values are already determined. If the current node is the root, then the search terminates with the optimal marginal MAP value (line 27). If $n$ is an OR node, then its parent $p$ is an AND node, and therefore $p$ updates its current value $v(p)$ by multiplication with the value of $n$ (line 29). If node $n$ was previously labeled optimal then the algorithm also saves in cache its value. An AND node $n$ propagates its value to its parent $p$ in a similar way, either by maximization or by summation depending on whether $p$ is labeled by a MAP or by a summation variable (lines 31-33). Finally, the current node $n$ is set to its parent $p$ (line 34), because $n$ was completely evaluated. Search continues either with a propagation step (if conditions are met) or with an expansion step. The algorithm can be easily instrumented to also recover the optimal solution tree $\hat{x}$ corresponding to the optimal marginal MAP value (see also Marinescu and Dechter (2009a) for additional details).

Our AOBB typically computes its heuristic $h(\cdot)$ using a weighted mini-bucket bounding scheme (see Section 3), which can be pre-compiled along the reverse order of a depth-first traversal of the valid pseudo-tree which is built along a constrained elimination order.

\subsubsection{BREADTH ROTATING AOBB (BRAOBB)}

Depth-first AND/OR branch and bound often lacks good anytime behavior because, during search, AOBB will solve to completion all but one independent subproblems rooted at an AND node. This behavior was first observed by Otten and Dechter (2011) in the context of pure MAP inference. Therefore, Breadth Rotating AOBB (BRAOBB) was introduced as an anytime depth-first AND/OR search scheme that rotates through different subproblems in a round-robin manner. Empirical evaluations showed that BRAOBB considerably improves AOBB's anytime behavior, and is better able to produce suboptimal solutions quickly and then gradually improve upon them. Without going into the details, the algorithm described in Otten and Dechter (2011) can be applied directly to marginal MAP queries by performing subproblem rotation to the MAP variables only.

\subsection{AOBF: Best-First AND/OR Search}

We next introduce a best-first search algorithm over the context-minimal AND/OR search graph (Algorithm 4). The algorithm belongs to the so-called AO* family (Nilsson, 1980). Like any AO*, AOBF maintains a partially explored AND/OR graph $C_{\mathcal{T}}$ and its current best partial solution tree $T^{\prime}$ that represents an optimal solution extension of $C_{\mathcal{T}}$ under the assumption that the tips $n$ of $C_{\mathcal{T}}$ are the terminal nodes with values given by the heuristic $h(n)$ (if labeled by a MAP variable) or by the corresponding conditioned likelihood (if labeled by a sum variable), respectively (see line 10). Initially, $C_{\mathcal{T}}$ contains the root node $s$ which is an OR node labeled by the root of the pseudo-tree $\mathcal{T}$. Then, at each iteration, the algorithm selects a non-terminal leaf node of the partial solution tree $T^{\prime}$ and expands it by generating its successors. The best partial tree is then recomputed by backward node value propagation, which sets the values of the leaves in $C_{\mathcal{T}}$ to its heuristic or conditioned likelihood values. The latter are calculated using procedure $\operatorname{eval}\left(\left.\mathcal{M}\right|_{T^{\prime}}\right)$ which solves exactly the summation sub-problem rooted by the respective sum variable. Clearly, we can use one of the best algorithms for summation subproblems such as the depth-first AND/OR search with full/adaptive 


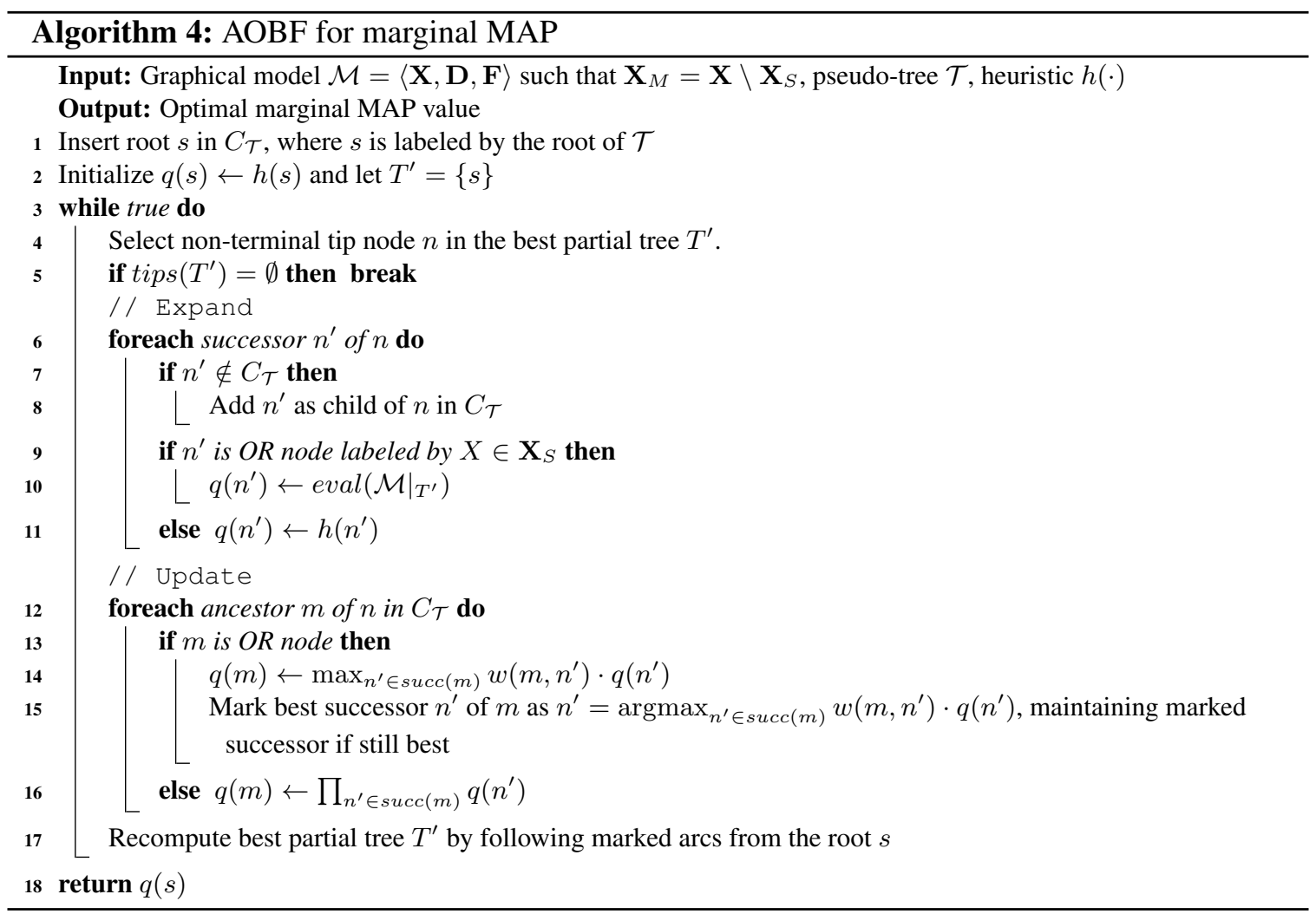

caching (Mateescu \& Dechter, 2007), and also allow sharing the summation sub-task computations via caching. Algorithm AOBF terminates when there are no leaf nodes in the best partial tree $T^{\prime}$. In this case $T^{\prime}$ represents the optimal MMAP assignment and its value is given by the updated value of the root node.

The complexity of AOBF is bounded by the size of the underlying context minimal search graph, and is therefore time and space $O\left(n \cdot k^{w_{c}}\right)$, where $w_{c}$ is the induced width along the valid pseudo-tree (i.e., the constrained induced width) (Marinescu \& Dechter, 2009a, 2009b).

\subsection{RBFAOO: Recursive Best-First AND/OR Search}

In practice, however, AOBF may require an enormous amount of memory, especially on difficult problem instances. We therefore developed a limited memory best-first search scheme based on the recursive best-first search idea (Korf, 1993). The algorithm is called Recursive Best-First AND/OR search with Overestimation (RBFAOO) and was shown to be very effective for pure MAP (Kishimoto \& Marinescu, 2014). Algorithm 5 presents the extension of RBFAOO to the marginal MAP task.

RBFAOO uses a local threshold control mechanism to explore the context minimal AND/OR search graph in a depth first-like manner (Korf, 1993). Let the q-value $q(n)$ be an upper bound of the solution cost at node $n$, and let $\theta(n)$ be the threshold at $n$ indicating the availability of a second best solution cost besides $q(n)$. RBFAOO keeps examining the subtree rooted at $n$ until either $q(n)<\theta(n)$ or the subtree rooted at $n$ is solved optimally. Therefore, it gradually grows the search space by updating the q-values of the internal nodes and unlike AOBF, re-expanding 


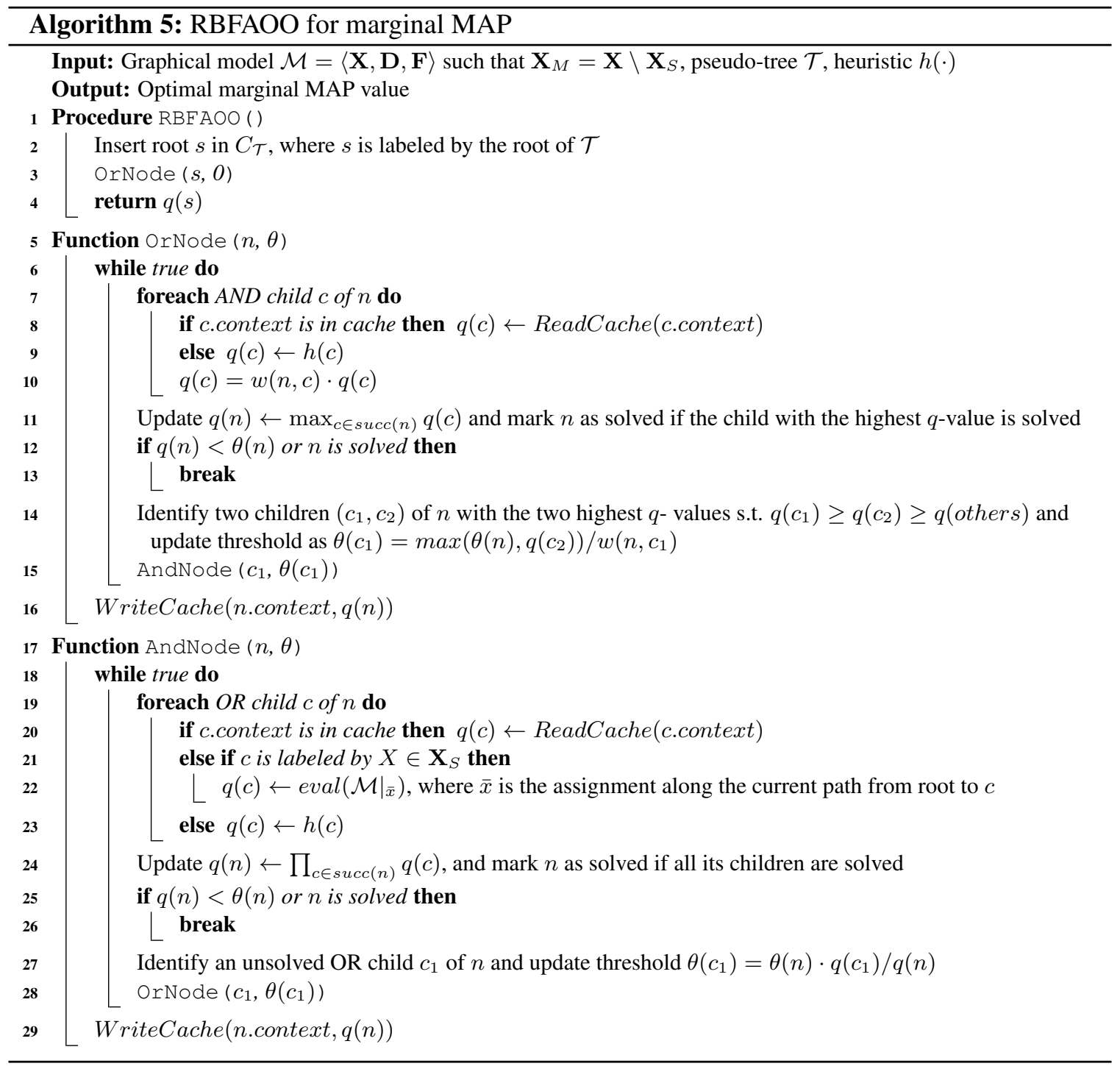

them. The algorithm may operate with linear space (no caching). However, it can also use a fixed size cache table to store some of the nodes (based on their contexts). When RBFAOO selects the next best child to expand, it examines it with a new threshold which is updated appropriately for OR and for AND nodes (see lines 14 and 27). Notice also that when expanding an AND node, the algorithm evaluates the conditional likelihood for all OR children that are labeled by sum variables using procedure $\operatorname{eval}(\cdot)$ (line 22). For efficiency, we implemented this step using the same recursive best-first approach but with the threshold set to $-\infty$ which essentially corresponds to depth-first AND/OR search with bounded cache.

If AOBF expands $O(N)$ nodes in the worst case, then RBFAOO expands $O\left(N^{2}\right)$ due to node re-expansions. Since $N$ is bounded by $O\left(n \cdot k^{w_{c}^{*}}\right)$, the time complexity of RBFAOO is bounded by $O\left(n^{2} \cdot k^{2 \cdot w_{c}^{*}}\right)$ but it can operate in linear space. However, in practice, by slightly overestimating the 


\begin{tabular}{|l||c|c|c|}
\hline Algorithm & Search Space & Heuristic & Variable Ordering \\
\hline \hline AOBB & AND/OR & WMB-MM(i) & Static \\
\hline BRAOBB & AND/OR & WMB-MM(i) & Static \\
\hline AOBF & AND/OR & WMB-MM(i) & Static \\
\hline RBFAOO & AND/OR & WMB-MM(i) & Static \\
\hline PARK & OR & Unconstrained join tree & Dynamic \\
\hline YUAN & OR & Unconstrained join tree & Static \\
\hline BBBTd & OR & MCTE(i) - unconstrained join tree & Dynamic \\
\hline BBBTi & OR & MCTE(i) - unconstrained join tree & Static \\
\hline OBB & OR & WMB-MM(i) & Static \\
\hline
\end{tabular}

Table 2: Marginal MAP search algorithms. Search spaces are either AND/OR search graph or OR search graph introduced in Section 2, and heuristic functions are defined in Section 3. Dynamic variable ordering schemes re-evaluate the heuristic function as search proceeds, whereas static schemes only evaluate it once before search.

node thresholds (by a small constant $\delta$ ), RBFAOO avoids such a high node re-expansion overhead (see also Kishimoto and Marinescu (2014) for more details).

\subsection{Experimental Results}

We next evaluate empirically the performance of the proposed depth-first and best-first AND/OR search algorithms as exact schemes for solving optimally the marginal MAP task. All competing algorithms were implemented in $\mathrm{C}++$, and the experiments were conducted on a cluster with 27 computing nodes. Each node has a dual-core $2.67 \mathrm{GHz}$ Intel Xeon X5650 CPU with $24 \mathrm{~GB}$ of RAM and was operated by a 64-bit Linux operating system. Each run of algorithms was allotted a 2-hour time limit and a 24 GB memory limit.

\subsubsection{Overview AND Methodology}

Algorithm Setup Table 2 shows all algorithms evaluated in the following experiments. We considered the AND/OR algorithms AOBB, AOBF, BRAOBB, and RBFAOO, respectively. They all use the weighted mini-bucket heuristic WMB-MM( $i$ ) (see Section 3) which has a single parameter called $i$-bound that controls the accuracy at the cost of using memory exponential in $i$. The hyperparameters of BRAOBB and RBFAOO are given as follows: we set the rotation limit to 1,000 for BRAOBB and the overestimation parameter to 1.0 for RBFAOO, respectively. The best-first AND/OR algorithms, AOBF and RBFAOO, use 4 GB of memory for storing the values of nodes in the AND/OR search graph. The depth-first AND/OR algorithms, AOBB and BRAOBB, use cache tables up to the total memory limit. We compare the AND/OR search algorithms with the OR search algorithms, PARK/YUAN and their generalizations BBBTd/BBBTi, respectively. We also evaluate the depth-first OR search algorithm with the WMB-MM(i) heuristic that we call OBB in order to compare the impact of using the AND/OR search space. For this purpose, we modified AOBB to explore an OR search space by enforcing a chain structure on the MAP variables in the guiding pseudo-tree.

Benchmarks The benchmark problem sets are derived from the Pascal2 competition problems (Elidan et al., 2012). They include (1) the grid domain which is a collection of random grid networks 


\begin{tabular}{|l|l||c|c|c|c|c|c|c|}
\hline \multicolumn{2}{|c|}{ Benchmark } & $\#$ inst & $n$ & $k$ & $w_{c}$ & $h_{c}$ & $w_{u}$ & $h_{u}$ \\
\hline \hline \multirow{2}{*}{ grid } & easy & 15 & $144-1156$ & $2-2$ & $16-52$ & $50-164$ & $15-49$ & $48-198$ \\
\cline { 2 - 9 } & hard & 60 & $144-1156$ & $2-2$ & $25-375$ & $42-421$ & - & - \\
\hline \multirow{2}{*}{ pedigree } & easy & 10 & $334-1289$ & $4-7$ & $35-237$ & $51-134$ & $15-29$ & $60-160$ \\
\cline { 2 - 9 } & hard & 40 & $334-1289$ & $4-7$ & $35-237$ & $63-259$ & - & - \\
\hline \multirow{2}{*}{ promedas } & easy & 10 & $453-1849$ & $2-2$ & $10-122$ & $42-174$ & $10-106$ & $43-157$ \\
\cline { 2 - 9 } & hard & 40 & $453-1849$ & $2-2$ & $11-490$ & $36-507$ & - & - \\
\hline
\end{tabular}

Table 3: Benchmark instances. \#. inst is the number of instances in each domain. We also distinguish easy and hard instances. The minimum and the maximum values from the set of problems are shown in the following parameters: $n$ is the number of variables, $k$ is the maximum domain size, $w_{c}$ is the constrained induced width, $h_{c}$ is the height of the pseudo-tree corresponding to the constrained elimination ordering. The unconstrained induced width, $w_{u}$ and pseudo-tree height, $h_{u}$ are also shown to highlight the difficulty of hard marginal MAP problem instances.

ranging from 12-by-12 to 34-by-34 grids, (2) the pedigree domain which is a collection of genetic linkage analysis problems that are related to the haplotyping task, and (3) the promedas domain which is a collection of Markov networks modeling an expert system for medical diagnosis tasks. Since the original problems from the Pascal 2 competition define pure MAP tasks, we generated synthetic marginal MAP problem instances from each MAP problem as follows. For each problem instance, we selected $m$ variables and marked them as MAP variables while the remaining ones were marked as summation variables, respectively. Specifically, we generated two types of marginal MAP instances with $m$ MAP variables for each network, easy and hard. The easy instances were generated by selecting the first $m$ variables from a breadth-first traversal of a pseudo-tree obtained from a join tree decomposition of the primal graph and the hard instances were generated by selecting the MAP variables uniformly at random. The easy instances were designed so that problem decomposition is maximized and the constrained and unconstrained elimination orders are relatively close to each other, thus having similar induced widths. In contrast, the hard instances tend to have very large constrained induced widths $w_{c}$ compared to the easy problem instances and to the unconstrained induced width $w_{u}$. In our experiments, $m$ is selected to be equal to $50 \%$ of the variables (see also Table 3 for more details). For consistency, all competing algorithms used the same constrained or unconstrained elimination ordering.

Measures of Performance From the evaluation results, we report the following metrics: (1) the CPU time in seconds for finding an optimal marginal MAP value from selected instances, (2) the number of summation problems evaluated until finding an optimal solution from selected instances, (3) the number of instances that returned an optimal solution from benchmark domains, and (4) the average CPU time for finding an optimal solution from benchmark domains. The first metric compares the CPU time for search algorithms that terminate with an optimal solution on an individual instance with varying strength of the heuristic functions controlled by the $i$-bound. The second metric compares the number of summation subproblems evaluated until finding an optimal solution on an individual instance with varying $i$-bounds, especially for the AND/OR search algorithms since 
the exact computation of summation subproblem imposes significant overheads to AND/OR search algorithms. The third metric visualizes the fraction of problem instances solved optimally given the total time and memory resources, and the last metric complements the third metric by comparing the average CPU time spent on finding an optimal solution from the common subset of benchmark domains that are solved by all AND/OR search based algorithms.

\subsubsection{THE CPU TIME FOR FINDING THE OPTIMAL SOLUTION}

Figure 2 shows the CPU time in seconds for finding the optimal solution on selected problem instances from the grid, pedigree and promedas domains, respectively. In each plot, we show only the algorithms that were able to prove optimality within the 2-hour time limit. Clearly, we see that the AND/OR search algorithms dominate all the OR search algorithms. The curves of AOBB, AOBF, BRAOBB, and RBFAOO lie below the curves of PARK, YUAN, BBBTd, BBBTi, and OBB at all $i$-bounds on all 6 instances. Note, the absence of a curve means that the algorithm could not terminate within the 2-hour time limit.

- PARK/YUAN failed to find an optimal solution for all problem instances except the easy pedigreel instance due to high unconstrained induced widths. The unconstrained induced width $w_{u}$ of each problem instance is $w_{u}=29$ for grid 75-21-5, $w_{u}=17$ for pedigreel, and $w_{u}=27$ for or_chain_$_{-} 4 f g$, respectively. Therefore, PARK/YUAN couldn't generate the unconstrained join-tree based heuristic for the grid 75-21-5 and or_chain_4fg instances because of the memory limit.

- BBBTd/BBBTi solved additional instances compared to PARK/YUAN, e.g., the easy or_chain_4fg was solved in 216 seconds and in 142 seconds by BBBTd and BBBTi, respectively. This is due to the use of the $\operatorname{MCTE}(i)$ heuristic that partitions the unconstrained join-tree with the $i$-bound.

- AOBB, AOBF, BRAOBB, and RBFAOO found an optimal solution in about 10 seconds with $i$-bound 16 or higher on these problem instances. Furthermore, on the hard or_chain_4fg instance, BBBTd and BBBTi found the optimal solution in 2863 seconds and 6832 seconds, respectively, while AOBB, AOBF, BRAOBB, and RBFAOO only spent 626 seconds, 293 seconds, 744 seconds, and 117 seconds, respectively, at the same $i$-bound 18 .

- RBFAOO found an optimal solution on the easy grid 75-21-5, the hard grid 75-21-5, the hard pedigree 1, and the hard or_chain_4fg instances with the smallest reported $i$-bounds (from 10 to 14) when other AND/OR search algorithms failed to find an optimal solution due to the time or memory limit.

We can see that algorithms AOBB and BRAOBB consistently dominate the OBB counterpart on all instances and at all reported $i$-bounds. Since OBB and AOBB are based on the same algorithm with the only difference being in how the search space is generated, we can conclude that the performance gain of AOBB and BRAOBB compared to OBB can be attributed to the compactness of AND/OR search space. When we compare OBB with other OR search algorithms we can see that OBB is the fastest OR search algorithm for finding an optimal solution. For example, OBB finds the optimal solution faster than BBBTd/BBBTi at the $i$-bound 18 or higher on the easy or_chain_4fg and hard or_chain_4fg instances. Furthermore, OBB is the only OR search algorithm that terminated within the 2 hour time limit on the easy grid 75-21-5 and the hard pedigree1 instances. Since OR search algorithms are only distinguished by the heuristic functions, we can also conclude that for 
(a) grid

\begin{tabular}{|c|c|c|c|c|c|c|c|c|c|}
\hline \multirow{2}{*}{$\begin{array}{c}\text { instance } \\
\left(n, f, k, w_{c}, w_{u}\right)\end{array}$} & \multirow[t]{2}{*}{ algorithm } & \multicolumn{2}{|c|}{$i=10$} & \multicolumn{2}{|c|}{$i=12$} & \multicolumn{2}{|c|}{$i=16$} & \multicolumn{2}{|c|}{$i=18$} \\
\hline & & time & nodes & time & nodes & time & nodes & time & nodes \\
\hline \multirow{9}{*}{$\begin{array}{l}\text { grid easy } \\
75-16-5 \text { I0 } \\
(256,256,2,22,21)\end{array}$} & PARK & \multicolumn{4}{|c|}{$\overline{167}$} & \multicolumn{4}{|c|}{1458} \\
\hline & YUAN & \multicolumn{4}{|c|}{29} & \multicolumn{4}{|c|}{17119} \\
\hline & $\overline{\text { BBBTd }}$ & oot & 302716 & 659 & 54520 & 42 & 17491 & 50 & 19584 \\
\hline & BBBTi & oot & 176156 & 2345 & 70590 & 85 & 30898 & 39 & 31424 \\
\hline & $\mathrm{OBB}$ & 1401 & 100538171 & 3 & 215404 & 0.48 & 7779 & 0.49 & 1733 \\
\hline & AOBB & 65 & 1919431 & 0.52 & 22334 & 0.41 & 3253 & 0.43 & 646 \\
\hline & BRAOBB & 7 & 566677 & 0.59 & 27771 & 0.35 & 2832 & 0.44 & 758 \\
\hline & AOBF & 7 & 212165 & 0.38 & 10687 & 0.27 & 2672 & 0.28 & 583 \\
\hline & RBFAOO & 1 & 290917 & 0.22 & 22279 & 0.16 & 2042 & 0.26 & 693 \\
\hline \multirow{9}{*}{$\begin{array}{l}\text { grid hard } \\
75-16-5 \mathrm{I} 1 \\
(256,256,2,82,21)\end{array}$} & PARK & \multirow{2}{*}{\multicolumn{4}{|c|}{$\frac{1415}{508}$}} & \multicolumn{4}{|c|}{5810} \\
\hline & YUAN & & \multicolumn{3}{|c|}{508} & \multicolumn{4}{|c|}{483065} \\
\hline & BBBTd & oot & 360097 & oot & 40291 & 4546 & 2389 & 2114 & 1607 \\
\hline & BBBTi & oot & 1009103 & oot & 115935 & 6288 & 3139 & oot & 3220 \\
\hline & OBB & oot & 220938105 & oot & 243066878 & oot & 319929239 & 85 & 4351922 \\
\hline & AOBB & oot & 398833504 & 632 & 37708909 & 5 & 340611 & 3 & 224719 \\
\hline & BRAOBB & 6964 & 380238949 & 324 & 18873588 & 5 & 350248 & 3 & 227999 \\
\hline & $\overline{\mathrm{AOBF}}$ & 728 & 15144899 & 721 & 13471576 & 10 & 249319 & 7 & 196812 \\
\hline & RBFAOO & 7161 & 553985425 & 309 & 29811222 & 7 & 784408 & 2 & 229745 \\
\hline
\end{tabular}

(b) pedigree

\begin{tabular}{|c|c|c|c|c|c|c|c|c|c|}
\hline \multirow{2}{*}{$\begin{array}{c}\text { instance } \\
\left(n, f, k, w_{c}, w_{u}\right)\end{array}$} & \multirow[t]{2}{*}{ algorithm } & \multicolumn{2}{|c|}{$i=10$} & \multicolumn{2}{|c|}{$i=12$} & \multicolumn{2}{|c|}{$i=16$} & \multicolumn{2}{|c|}{$i=18$} \\
\hline & & time & nodes & time & nodes & time & nodes & time & nodes \\
\hline \multirow{9}{*}{$\begin{array}{l}\text { pedigree easy } \\
\text { pedigree } 33 \mathrm{I} 0 \\
(798,798,4,28,24)\end{array}$} & PARK & \multicolumn{4}{|c|}{ oom } & \multicolumn{4}{|c|}{ oom } \\
\hline & YUAN & \multicolumn{4}{|c|}{ oom } & \multicolumn{4}{|c|}{ oom } \\
\hline & BBBTd & oot & 208815 & oot & 145663 & oot & 52744 & oot & 43948 \\
\hline & BBBTi & oot & 246582 & oot & 209620 & oot & 120843 & oot & 50220 \\
\hline & OBB & oot & 72686306 & 3584 & 31265034 & 726 & 5896706 & 745 & 527116 \\
\hline & $\mathrm{AOBB}$ & 20 & 877731 & 7 & 328531 & 4 & 107914 & 5 & 25357 \\
\hline & BRAOBB & 33 & 1498544 & 11 & 532770 & 5 & 157469 & 7 & 38222 \\
\hline & AOBF & 14 & 473329 & 7 & 212385 & 3 & 63495 & 4 & 17158 \\
\hline & RBFAOO & 10 & 1082112 & 4 & 450426 & 2 & 107853 & 4 & 29505 \\
\hline \multirow{9}{*}{$\begin{array}{l}\text { pedigree hard } \\
\text { pedigree } 33 \mathrm{I} 3 \\
(798,798,4,90,24)\end{array}$} & PARK & \multicolumn{4}{|c|}{ oom } & \multicolumn{4}{|c|}{ oom } \\
\hline & YUAN & \multicolumn{4}{|c|}{ oom } & \multicolumn{4}{|c|}{ oom } \\
\hline & BBBTd & oot & 129028 & oot & 50439 & oot & 9883 & oot & 7514 \\
\hline & BBBTi & oot & 17291423 & oot & 11827308 & oot & 2208894 & oot & 15783 \\
\hline & OBB & oot & 90882192 & oot & 88027605 & oot & 92162950 & oot & 108961101 \\
\hline & AOBB & oot & 371082807 & oot & 398810432 & 3064 & 194413004 & 334 & 20981934 \\
\hline & BRAOBB & oot & 252364162 & oot & 274187876 & oot & 274849031 & 700 & 32731707 \\
\hline & AOBF & oom & 17448194 & oom & 16341293 & oom & 15891613 & oom & 11957878 \\
\hline & RBFAOO & oot & 653477484 & 3751 & 333264049 & 880 & 86995051 & 55 & 6515774 \\
\hline
\end{tabular}

(c) promedas

\begin{tabular}{|c|c|c|c|c|c|c|c|c|c|}
\hline \multirow{2}{*}{$\begin{array}{c}\text { instance } \\
\left(n, f, k, w_{c}, w_{u}\right)\end{array}$} & \multirow{2}{*}{ algorithm } & \multicolumn{2}{|c|}{$i=10$} & \multicolumn{2}{|c|}{$i=12$} & \multicolumn{2}{|c|}{$i=16$} & \multicolumn{2}{|c|}{$i=18$} \\
\hline & & time & nodes & time & nodes & time & nodes & time & nodes \\
\hline \multirow{9}{*}{$\begin{array}{l}\text { promedas easy } \\
\text { or chain8 fg I0 } \\
(1044,1055,2,63,56)\end{array}$} & PARK & \multicolumn{4}{|c|}{ oom } & \multicolumn{4}{|c|}{ oom } \\
\hline & YUAN & \multicolumn{4}{|c|}{ oom } & \multicolumn{4}{|c|}{ oom } \\
\hline & BBBTd & oot & 123551 & oot & 28609 & 3191 & 17816 & oot & 13992 \\
\hline & BBBTi & oot & 1202749 & oot & 85250 & oot & 25481 & oot & 13975 \\
\hline & OBB & oot & 45854883 & oot & 44042781 & oot & 24204379 & oot & 21909874 \\
\hline & AOBB & oot & 430376779 & oot & 359556370 & oot & 391666636 & 1583 & 108529398 \\
\hline & BRAOBB & oot & 359396003 & oot & 312111365 & 5288 & 272736813 & 758 & 57647876 \\
\hline & $\mathrm{AOBF}$ & oom & 16843900 & oom & 17038664 & oom & 17191924 & 510 & 15923902 \\
\hline & RBFAOO & 6425 & 555895245 & 1268 & 126337093 & 682 & 78371234 & 152 & 21786146 \\
\hline \multirow{9}{*}{$\begin{array}{l}\text { promedas hard } \\
\text { or chain4 fg I4 } \\
(691,701,2,100,27)\end{array}$} & PARK & \multicolumn{4}{|c|}{ oom } & \multicolumn{4}{|c|}{ oom } \\
\hline & YUAN & \multicolumn{4}{|c|}{ oom } & \multicolumn{4}{|c|}{ oom } \\
\hline & BBBTd & 3496 & 4178 & 607 & 2650 & 261 & 3009 & 591 & 2031 \\
\hline & BBBTi & 4319 & 31507 & 4798 & 12221 & 2463 & 3699 & 1545 & 3295 \\
\hline & OBB & oot & 54940954 & oot & 60336495 & oot & 80233378 & oot & 84429447 \\
\hline & AOBB & oot & 388478093 & oot & 403472921 & 497 & 30889027 & 55 & 4205131 \\
\hline & BRAOBB & 3320 & 199196858 & 2127 & 147963142 & 154 & 12173651 & 33 & 2754174 \\
\hline & AOBF & 630 & 17599487 & 550 & 17612588 & 100 & 3276123 & 44 & 1505211 \\
\hline & RBFAOO & 215 & 29608915 & 326 & 44855073 & 26 & 4260266 & 9 & 1443645 \\
\hline
\end{tabular}

Table 4: CPU time in seconds and number of nodes expanded for solving selected problem instances. Time limit 2 hours, memory limit 24GB. 'oot' and 'oom' stand for out-of-time and out-ofmemory, respectively. 

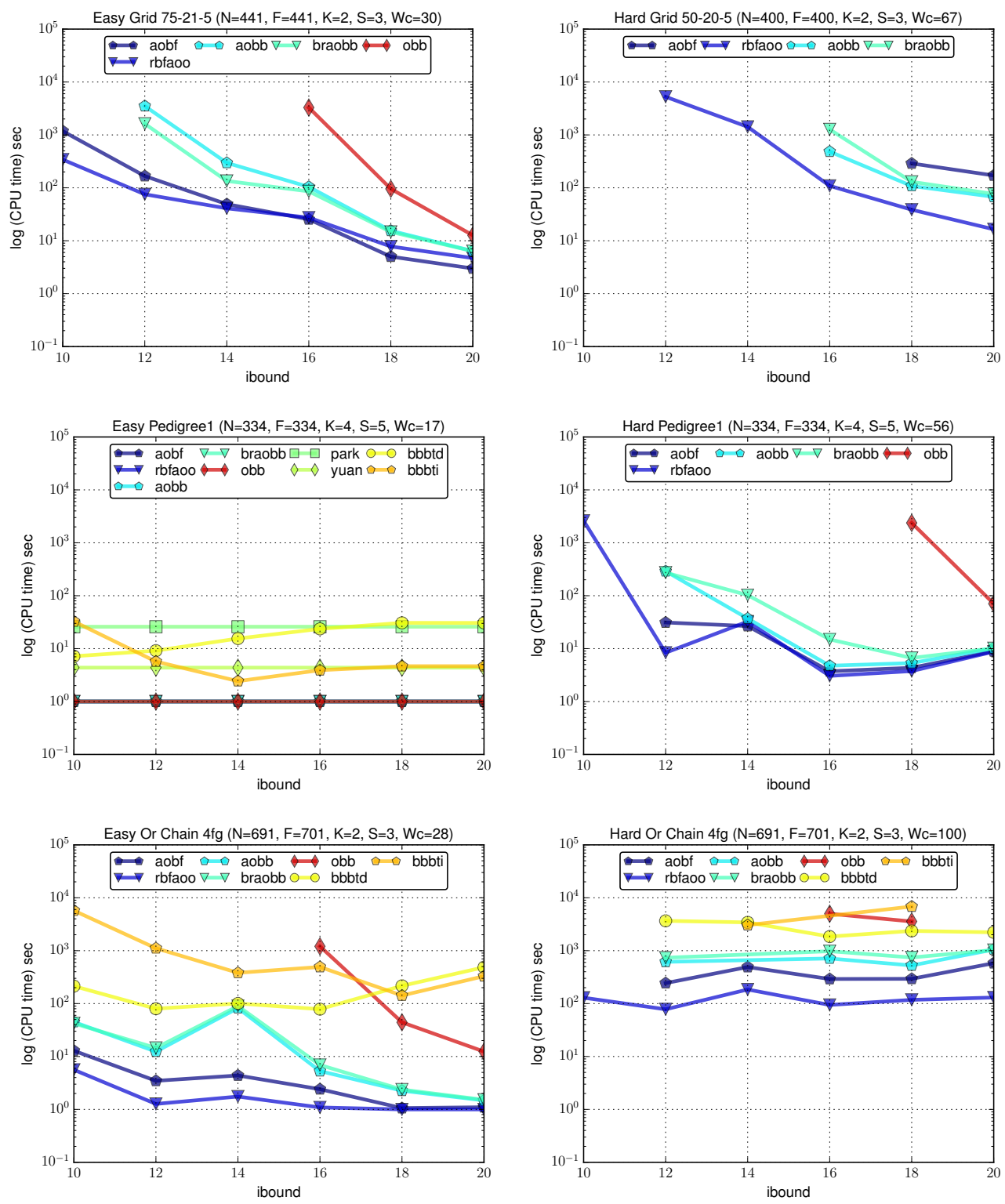

Figure 2: The CPU time in seconds from 6 problem instances. The horizontal axis is the i-bound of WMB-MM(i) heuristic for AND/OR search algorithms and MCTE(i) heuristic for OR search algorithms. The vertical axis is the CPU time in log scale. The missing data points indicate a search algorithm failed to find the optimal solution in time limit of 2 hours. PARK/YUAN are shown only in the easy pedigree 1 instance since both failed to find the optimal solution for all other instances in the time limit, and BBBTi/BBBTd are also shown in the easy pedigree 1 instance and promedas instances.

the same $i$-bound value the WMB-MM( $i$ ) heuristic is tighter than the $\operatorname{MCTE}(i)$ heuristic used by BBBTd/BBBTi. 
When comparing the AND/OR search algorithms, we can conclude that the best-first search algorithms perform better than the depth-first search ones on all problem instances and at all $i$-bounds according to the current metric. Specifically, RBFAOO the variant that operates within limited memory, emerges as the best performing exact AND/OR search algorithm. Table 4 reports additional results on individual problem instances from the 3 benchmark domains. The results display similar patterns as before, namely the AND/OR search algorithms dominate the OR search algorithms.

\subsubsection{THE NUMBER OF CONDITIONAL LIKELIHOOD EVALUATIONS}

Figure 3 shows the number of conditional likelihood evaluations by the AND/OR search algorithms from 6 problem instances at varying $i$-bounds. Note that we only show the number of conditional likelihood evaluations when a search algorithm found the optimal solution. Thus, the missing data points in the plot indicates a search algorithm couldn't find the optimal solution within the 2-hour time limit. For example:

- On the easy grid 75-21-5 instance with $i$-bound 18, AOBF, RBFAOO, AOBB and BRAOBB evaluated 282, 173, 3106, and 1924 summation subproblems, respectively. The trend is similar on the hard grid 75-21-5 instance as well.

- On the hard grid 50-20-5 instance with $i$-bound 18, AOBF, RBFAOO, AOBB and BRAOBB evaluated 226474, 55355, 481760, and 572050 summation subproblems.

- On the hard pedigree 9 instance with $i$-bond 18, AOBF, RBFAOO, AOBB and BRAOBB evaluated 2985, 4838, 6410, and 21632 summation subproblems.

We can see that AOBF and RBFAOO consistently evaluate a smaller number of summation problems than AOBB and BRAOBB. It is important to note that RBFAOO has a much smaller computational overhead compared with AOBF which often translates into a much higher node expansion rate per second than that of AOBF. This means that RBFAOO is able to reach the most promising region of the search space (containing the optimal solution) quicker than AOBF which explains the reduced number of conditional likelihood evaluations of RBFAOO. We discuss next the two major sources of AOBF's significant additional overhead. First, when expanding the current node $n$, AOBF may need to update the node values of all $n$ 's ancestors all the way up to the root, whereas RBFAOO may only update the q-value of $n$ 's parent. Second, AOBF needs to maintain in memory the entire explicated search graph and this may be difficult especially when the memory used approaches the 24GB limit. In contrast, RBFAOO conducts the search in a depth-first like manner and uses only 4GB of RAM for its cache table. We also observe that higher $i$-bounds lead to fewer summation evaluations for all search algorithms because of improved heuristics and more effective pruning.

\subsubsection{THE NUMBER OF INSTANCES THAT RETURNED AN OPTIMAL SOLUTION}

Figure 4 provides aggregated results showing the number of instances on which the algorithms returned an optimal solution for all 3 problem domains combining easy and hard problem instances. We observe that the AND/OR search algorithms solved a larger number of instances than the OR search algorithms on all domains. Moreover, the best-first AND/OR search algorithms, AOBF and RBFAOO, solved more instances than the depth-first AND/OR search algorithms, AOBB and BRAOBB, respectively. 

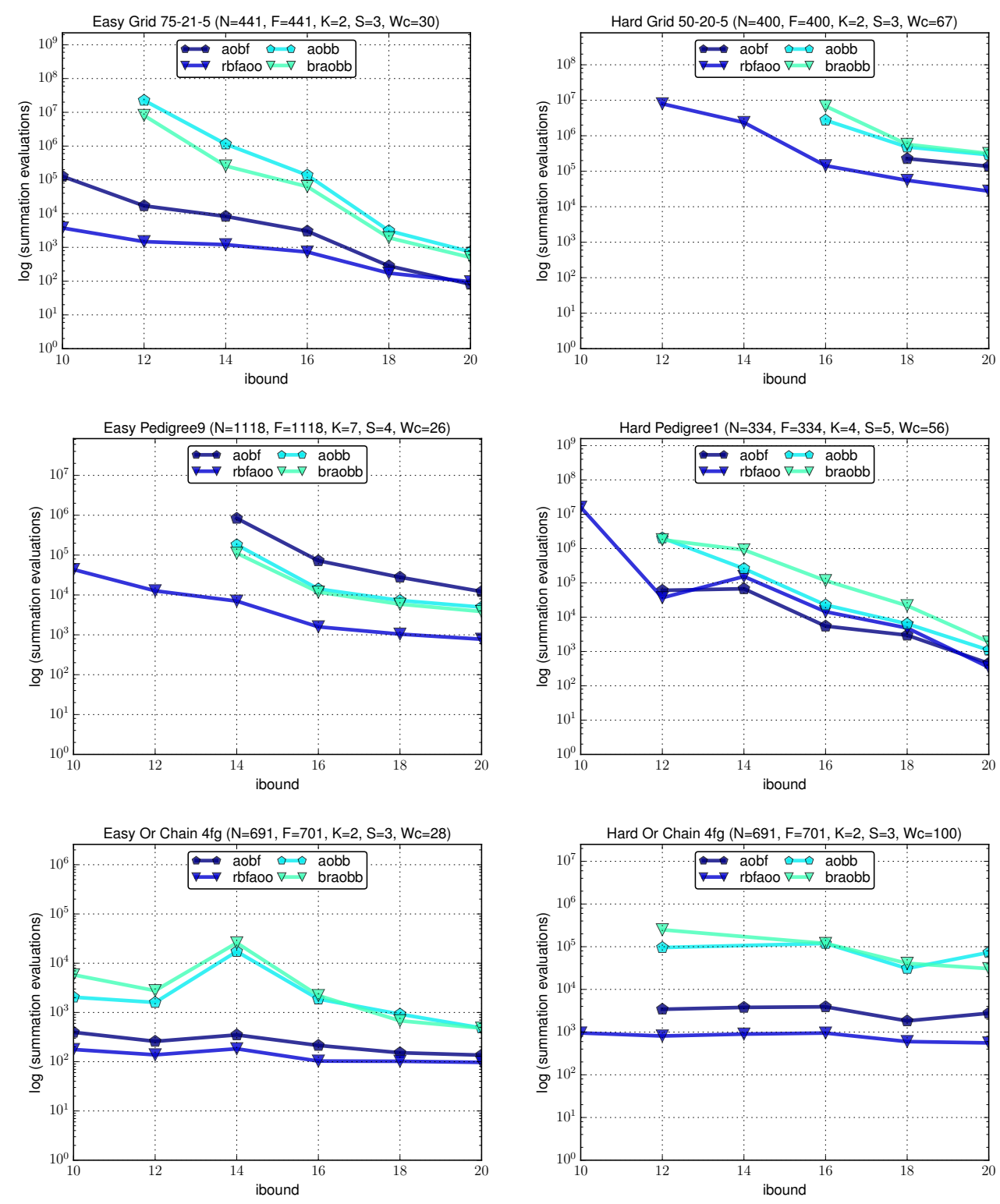

Figure 3: The number of conditional likelihood evaluations of AND/OR search algorithms from 6 problem instances. The horizontal axis is the i-bound of WMB-MM(i) heuristic for AND/OR search algorithms and MCTE(i) heuristic for OR search algorithms. The vertical axis is the number of summation problems solved until finding the optimal solution within 2 hour time limit.

- On the grid domain with $i$-bound 20, AOBF, RBFAOO, AOBB, BRAOBB, OBB, BBBTd, and BBBTi solved $76 \%, 79 \%, 73 \%, 76 \%, 55 \%, 28 \%$, and $23 \%$ of the problem instances, respectively. 

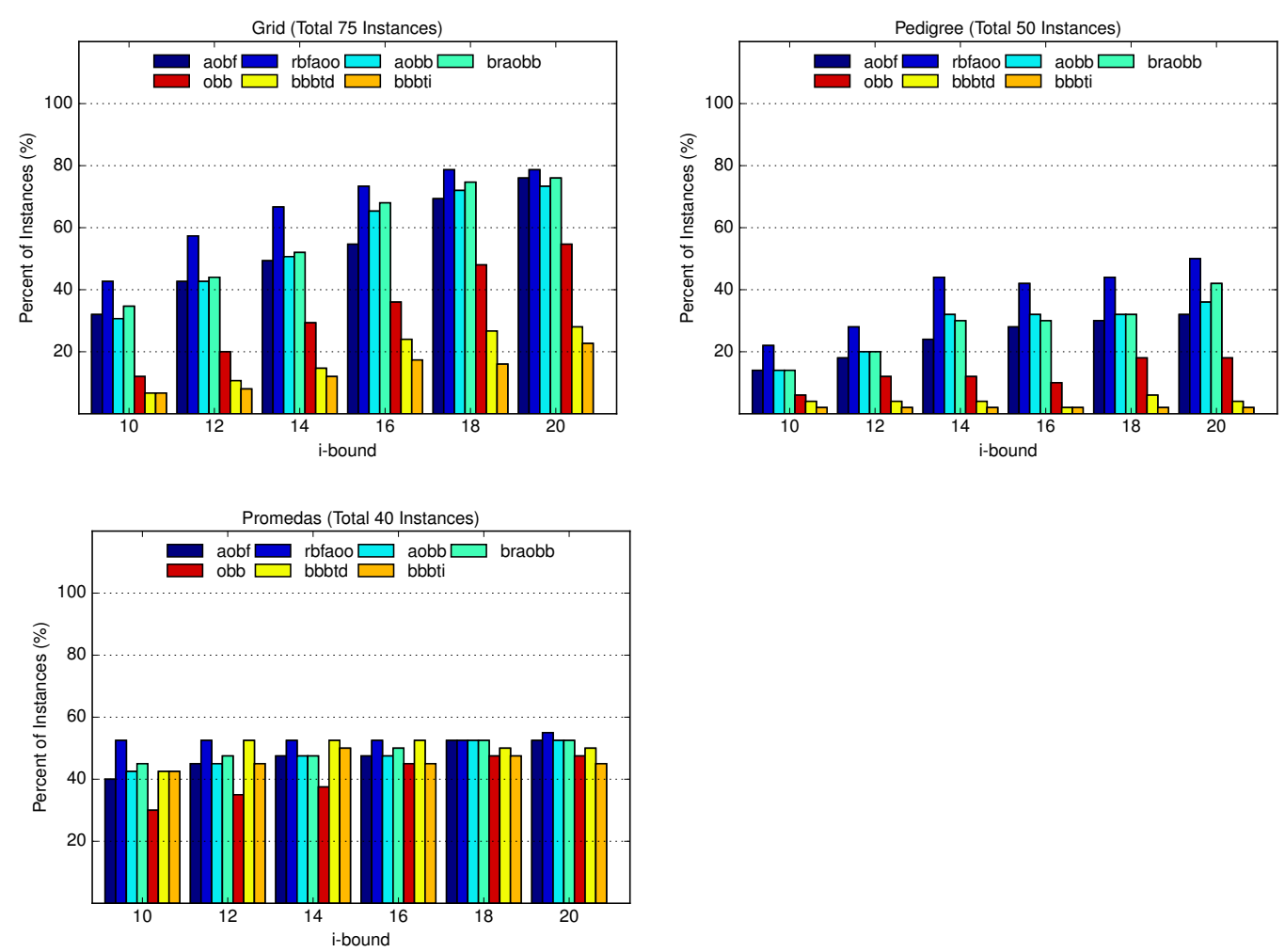

Figure 4: The number of instances returned with the optimal solution from 3 problem domains. Easy and hard problem instances are combined into a single group. The horizontal axis is the i-bound of WMB-MM(i) heuristic function and the vertical axis is the normalized count on the instances returned with the optimal solution in 2 hour time limit. AND/OR search algorithms solved more instances than OR search algorithms, and RBFAOO solved the largest number of instances at all domains over all i-bounds.

- On pedigrees with $i$-bound 20, AOBF, RBFAOO, AOBB, BRAOBB, OBB, BBBTd, and BBBTi solved $32 \%, 50 \%, 36 \%, 42 \%, 18 \%, 4 \%$, and $2 \%$ following the similar trend as in the grid domain.

- On promedas with $i$-bound 20, AOBF, RBFAOO, AOBB, BRAOBB, OBB, BBBTd, and BBBTi solved 52\%, 55\%, 53\%, 53\%, 47\%, 50\%, and 40\%, showing that RBFAOO still performs the best but the difference between AND/OR search algorithms and OR search algorithms are less significant than on the other two domains.

Clearly, RBFAOO is the best performing algorithm on all domains and at all reported $i$-bounds. Comparing AOBF and AOBB, both algorithms solved a similar number of instances in the 2-hour time limit and the 24 GB memory limit, while BRAOBB solved slightly fewer instances than AOBB because of the computational overhead associated with rotating over the independent subproblems in the AND/OR search space. BBBTd and BBBTi solved the smallest number of problem instances across all domains and both failed to solve even a single instance from the hard pedigree 

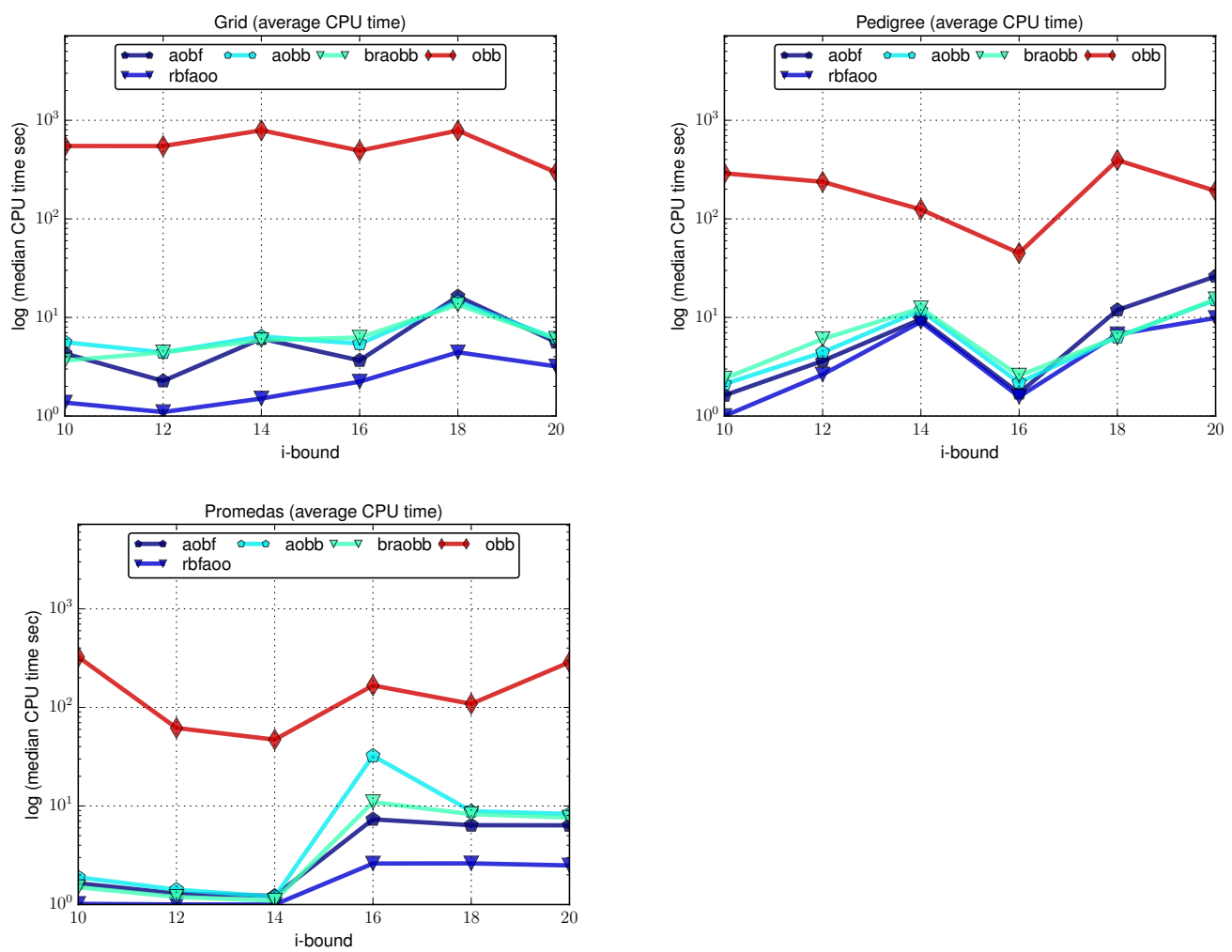

Figure 5: The average CPU time for finding the optimal solutions in a benchmark. The horizontal axis is the i-bound of WMB-MM(i) heuristic function and the vertical axis is the average CPU time for finding the optimal solution in log scale. The average time was computed from the common problem instances that are solved by all four algorithms in two hours. The best-first search spent less time than depth-first search. RBFAOO is the fastest algorithm across all problem domains and i-bounds.

instances. The performance of OBB lies between AND/OR search algorithms and the other OR search algorithms guided by unconstrained join-tree based heuristics, thus demonstrating on one hand the effectiveness of the WMB-MM $(i)$ heuristics compared to the MCTE $(i)$ with unconstrained order, and the compactness of the AND/OR search space versus the OR search space on the other hand.

\subsubsection{THe AVERAge CPU TIME}

Since the previous metric only considers the number of solvable instances (thus providing the provable optimal solution), we report in Figure 5 the average CPU time in seconds for finding the optimal solution, for problem instances solved by all four AND/OR search algorithms to avoid bias: 40 grid instances, 9 pedigree instances, and 19 promedas instances were solved optimally by all four algorithms with $i$-bound 20 . 
- On the grid domain with $i$-bound 20, the average CPU times of AOBF, RBFAOO, AOBB, BRAOBB, and OBB search algorithms are 5.61 seconds, 3.18 seconds, 6.18 seconds, 6.07 seconds, and 296.11 seconds, respectively. RBFAOO and AOBF solved problem instances faster than BRAOBB and AOBB, and OBB spent significantly longer time compared to the AND/OR search algorithms.

- On pedigrees with $i$-bound 20, the average CPU times of AOBF, RBFAOO, AOBB, BRAOBB, and OBB search algorithms are 26.13 seconds, 9.87 seconds, 15.09 seconds, 15.26 seconds, and 192.05 seconds, respectively. We see again a similar trend as before.

- On promedas with $i$-bound 20, the average CPU times of AOBF, RBFAOO, AOBB, BRAOBB, and OBB search algorithms are 6.36 seconds, 2.49 seconds, 8.30 seconds, 7.63 seconds, and 287.27 seconds, respectively.

In summary, for exact algorithms, we conclude that RBFAOO is overall the best exact search algorithm. We also see that AOBF can solve problem instances faster than AOBB and BRAOBB at higher $i$-bounds, while AOBB and BRAOBB are competitive with AOBF at smaller $i$-bounds.

\section{Anytime Search Algorithms for Marginal MAP}

In the previous section we introduced the main exact search schemes traversing the AND/OR space for marginal MAP. These schemes have obvious shortcomings. Best-first search provides a solution only at termination. Depth-first search is more flexible with anytime capabilities and seems more tuned with our goal of yielding anytime performance as well as bounds that can be tightened with time. In this section we show how we can move towards both these goals by building upon the complementary properties of best-first and depth-first search. We first explore the potential of the well-known principle of weighted search that converts best-first search algorithms into anytime search schemes (Section 5.1). This scheme was previously explored for pure MAP (Flerova et al., 2017), and we will show in the sequel how it can be extended to marginal MAP. Subsequently, (Section 5.2), we will present an alternative anytime approach that combines the best-first and depth-first principles into hybrid search schemes. Both methods provide anytime confidence intervals in the form of improving upper and lower bounds on the optimum.

\subsection{Weighted Best-First AND/OR Search}

Weighted best-first AND/OR search was introduced recently as an effective alternative to anytime depth-first search schemes for pure MAP tasks (Flerova et al., 2017). These algorithms were evaluated on a wide range of benchmark problems and were shown to be highly competitive with Breadth Rotating AOBB search (Otten \& Dechter, 2011), one of the best performing anytime MAP solvers.

We first note that in this section (and only in this one) we will convert the marginal MAP task into a minimization task, because weighted heuristic schemes are normally, and more naturally described in the context of min-sum tasks. We show next how to transform Equation 1 into a minimization problem. Let $F_{M}$ be the subset of functions whose scopes are subsumed by MAP variables only, i.e., $F_{M}=\left\{\alpha: \mathbf{X}_{\alpha} \subseteq \mathbf{X}_{M}\right\}$. Then, we have that: 


$$
\begin{aligned}
\mathbf{x}^{*} & =\underset{x_{M}}{\operatorname{argmin}}-\log \left(\sum_{\mathbf{x}_{s}} \prod_{\alpha \in F} \psi_{\alpha}\left(\mathbf{x}_{\alpha} \mid x_{M}\right)\right) \\
& =\underset{x_{M}}{\operatorname{argmin}}-\log \left(\sum_{\mathbf{X}_{s}} \prod_{\alpha \in F_{M}} \psi_{\alpha}\left(\mathbf{x}_{\alpha} \mid x_{M}\right) \cdot \prod_{\alpha \in F \backslash F_{M}} \psi_{\alpha}\left(\mathbf{x}_{\alpha} \mid x_{M}\right)\right) \\
& =\underset{x_{M}}{\operatorname{argmin}}-\log \left(\prod_{\alpha \in F_{M}} \psi_{\alpha}\left(\mathbf{x}_{\alpha} \mid x_{M}\right) \cdot \sum_{\mathbf{X}_{s}} \prod_{\alpha \in F \backslash F_{M}} \psi_{\alpha}\left(\mathbf{x}_{\alpha} \mid x_{M}\right)\right) \\
& =\underset{x_{M}}{\operatorname{argmin}}-\log \left(\prod_{\alpha \in F_{M}} \psi_{\alpha}\left(\mathbf{x}_{\alpha} \mid x_{M}\right)\right)-\log \left(\sum_{\mathbf{X}_{s}} \prod_{\alpha \in F \backslash F_{M}} \psi_{\alpha}\left(\mathbf{x}_{\alpha} \mid x_{M}\right)\right) \\
& =\underset{x_{M}}{\operatorname{argmin}} \sum_{\alpha \in F_{M}}-\log \psi_{\alpha}\left(\mathbf{x}_{\alpha} \mid x_{M}\right)-\log \left(\sum_{\mathbf{X}_{s}} \prod_{\alpha \in F \backslash F_{M}} \psi_{\alpha}\left(\mathbf{x}_{\alpha} \mid x_{M}\right)\right) \\
& =\underset{x_{M}}{\operatorname{argmin}} \sum_{\alpha \in F_{M}} \theta_{\alpha}\left(\mathbf{x}_{\alpha} \mid x_{M}\right)+\phi\left(x_{M}\right)
\end{aligned}
$$

It is easy to see that $\phi\left(x_{M}\right)$ corresponds to the summation subproblem conditioned on the assignment $x_{M}$ to the MAP variables $\mathbf{X}_{M}$. Therefore, algorithms AOBF and RBFAOO can be modified in a straightforward manner to solve Equation 2 by replacing maximization with minimization at the OR nodes labeled by MAP variables, and multiplication with summation at their AND children, respectively. We also note that for each of the conditioned summation subproblems the algorithms consider the negative log of the respective value.

Yet, note that when the arc-costs are larger than 1, the negative log-transformation yields negative numbers (e.g. Markov networks), which are not suitable for the min-sum formulation. It may be possible to handle this by normalizing by a large constant. Since our benchmarks are all based on Bayesian networks we did not encounter this problem and did not explore nor experimented with this issue and we leave this for future work.

\subsubsection{WEIGHTED AOBF AND RBFAOO}

The fixed-weighted version of the AOBF and RBFAOO algorithms can be obtained by multiplying the heuristic function $h(n)$ of a node $n$ in the AND/OR search graph by a weight $\alpha>1$ (i.e., substituting $h(n)$ by $\alpha \cdot h(n))$. This would involve only the portion of the search space that corresponds to the MAP variables. If $h(n)$ is admissible, which is the case for mini-bucket heuristics, then the cost of the solution obtained by weighted AOBF (or weighted RBFAOO) is $\alpha$-optimal, namely it is guaranteed

to be within a factor $\alpha$ from the optimal one. These schemes extend well known approaches such as WA* (Pohl, 1970) and WAO* (Chakrabarti, Ghose, \& Sarkar, 1987) to the marginal MAP query.

\subsubsection{Iterative Weighted AOBF With REPAIRS}

Since weighted AOBF yields $\alpha$-optimal solutions, it can then be extended into an iterative anytime scheme that we call WAOBF (Algorithm 6), by decreasing the weight from one iteration to the next (until it becomes 1). Specifically, WAOBF starts by running weighted AOBF with an initial weight 

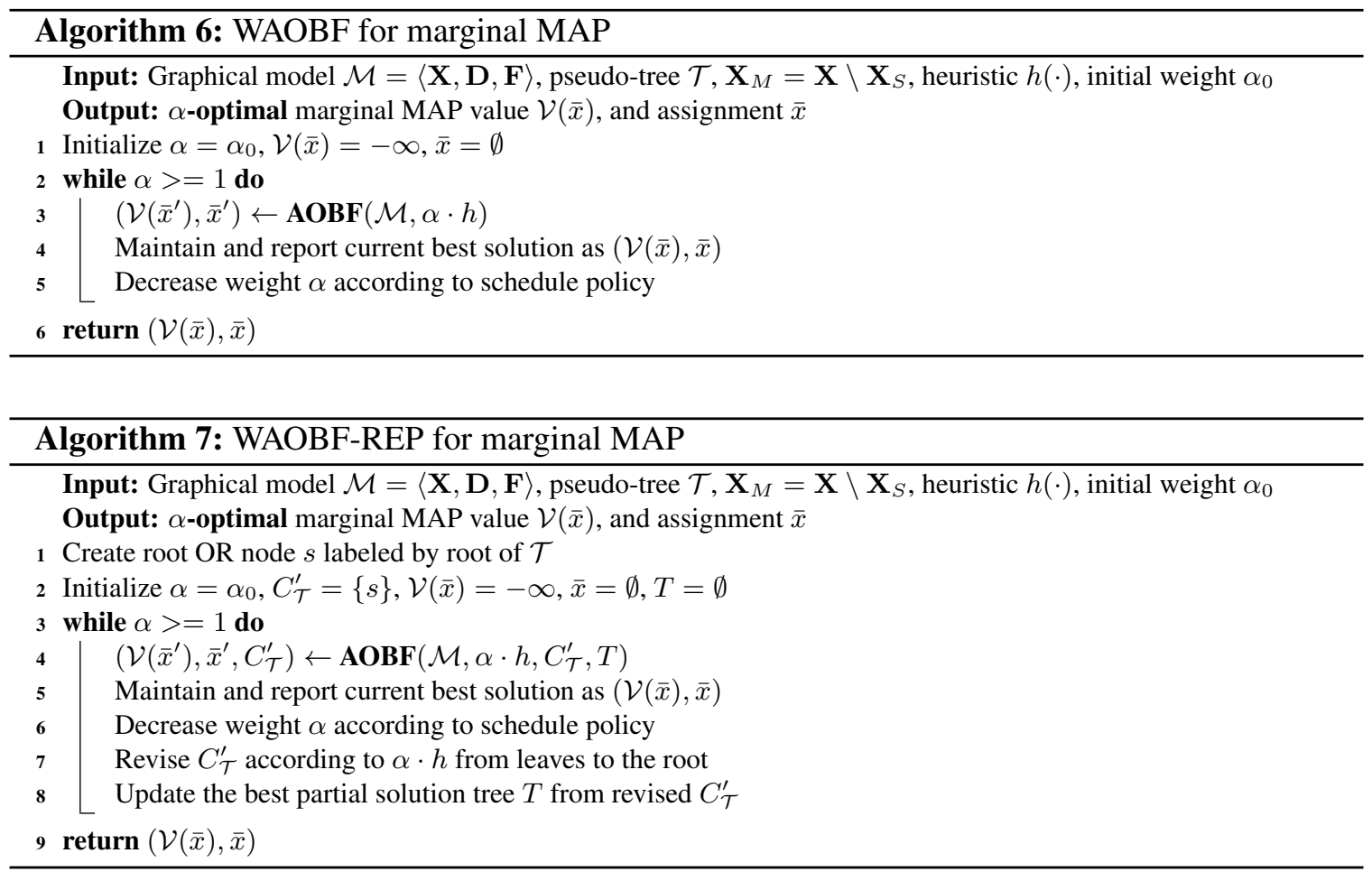

$\alpha_{0}$ until it finds a suboptimal solution. It then restarts the search with a decreased weight using a weight update schedule policy such as $\alpha_{i+1}=\sqrt{\alpha_{i}}$. Clearly, different schedules can be used, however, the latter proved quite effective in practice when solving a MAP query (Flerova et al., 2017). Notice that the algorithm also outputs a $\alpha$-optimality guarantee for the smallest $\alpha$ it reached.

Running WAOBF afresh at each iteration seems redundant however. Therefore, we introduce another anytime scheme, called WAOBF-REP, that is based on the anytime repairing AOBF approach for pure MAP (Flerova et al., 2017). The algorithm is capable of reusing information from previous iterations. WAOBF-REP is described in Algorithm 7. As noted, it does not discard the explicated AND/OR search graph $C_{\mathcal{T}}^{\prime}$ after finishing the $i$-th iteration but rather revises the node values in $C_{\mathcal{T}}^{\prime}$ from leaves to the root node according to the newly inflated heuristic $\alpha_{i+1} \cdot h(n)$. Then, search restarts with the revised best partial solution tree.

\subsubsection{ITERATIVE WEIGHTED RBFAOO}

Finally, an anytime weighted RBFAOO, called WRBFAOO, is obtained by replacing the call to AOBF in line 3 of Algorithm 6 with a call to RBFAOO, respectively.

\subsection{Hybrid Best+Depth-First AND/OR Search}

While weighted best-first search schemes turned out to be favorable for marginal MAP compared with depth-first search, as we will show in our experiments (see also Lee et al. (2016)), their anytime performance seems quite sensitive to the initial weight and to the weight update schedule. In particular: (i) the algorithms sometimes spend significant computational resources (time, memory) struggling to find even the first suboptimal solution; (ii) depending on the weight update schedule, 
either very few solutions are generated or there is no improvement in the solution quality; (iii) most critically, the sub-optimality bound produced by the weight is often very loose.

We now explore schemes that combine best-first and depth-first search into best+depth hybrid anytime search algorithms. These algorithms provide anytime upper and lower bounds on the optimal marginal MAP value that can be used to better gauge the solution quality during search, and these bounds tighten with time.

\subsubsection{NOTATIONS}

Let $C_{\mathcal{T}}^{\prime}$ denote the explicated context-minimal AND/OR search graph relative to pseudo-tree $\mathcal{T}$. Note that in this case $C_{\mathcal{T}}^{\prime}$ is defined over the MAP variables only. Each node $n \in C_{\mathcal{T}}^{\prime}$ maintains two values $q(n)$ and $l(n)$, respectively. The quantity $q(n)$ represents an upper bound provided by the heuristic evaluation function at the node $n$ (i.e., the weighted mini-bucket value), while $l(n)$ is the cost of the current best solution found below node $n$, and is therefore a lower bound on the best solution in the search space below $n$. We use $\mathcal{U}$ and $\mathcal{L}$ to denote the current best global upper and lower bounds on the optimal MMAP of the root node. For node $n \in C_{\mathcal{T}}^{\prime}, \operatorname{ch}(n)$ denote its children in $C_{\mathcal{T}}^{\prime}$, while $w_{(n, m)}$ is the weight labeling the arc $n \rightarrow m$ in $C_{\mathcal{T}}^{\prime}$. Algorithm 8 describes the node expansion (EXPAND $(n)$ ) and node values update (UPDATE $(n)$ ) procedures during search. Clearly, the $q$ - and $l$-values are updated bottom-up based on the corresponding values of their children in the search graph. Note that during the update of $q$-values, we also mark with a $\star$ symbol the arc corresponding to the best child $m^{\prime}$ of an OR node $n$.

\subsubsection{LAOBF: Best-First AND/OR SEARCH with DEPTH-FIRST LOOKAHEADS}

As noted before, the rationale behind best+depth based search is to alternate in some manner between the two styles of search space exploration, where the best-first component progresses towards improved upper bounds and depth-first progresses towards improved potential solutions and their accompanying lower bounds.

A simple way to augment best-first search with a mechanism that generates solutions is to do explicit depth-first lookahead dives under some nodes in the best-first search frontier (Stern, Kulberis, Felner, \& Holte, 2010). Our first best+depth hybrid, Best-First AND/OR Search with Depth-First Lookaheads (LAOBF), is described in Algorithm 9. We elaborate on these dives next.

DFS and BFS Iterations Let $T_{b}$ be the current best partial solution tree of the best-first search scheme, $\operatorname{tips}\left(T_{b}\right)$ be the set of tip nodes of $T_{b}$, and $m$ be one of these tips. The algorithm performs a depth-first dive at the subtree rooted at $m$, recording the conditioned lower bound found as $l(m)$. This is accomplished by the dfs-lookahead $(m)$ function in line 8. Once all the tips of $T_{b}$ are explored in such a depth-first manner, a global lower bound $\mathcal{L}$ of $T_{b}$ can be obtained by multiplying the generated lower bounds $l(m)$ by their corresponding arc costs in $T_{b}$ (line 10). Once such a full dive is accomplished, best-first search takes over as usual, expanding a tip node $n$ from the current $T_{b}$, updating the $q$-values of $n$ 's ancestors, and selecting the next best partial solution tree. Then, a new depth-first dive can be performed from all tip nodes, and so on. The updated $q$-value of the root node provides an anytime (and often improved) upper bound $\mathcal{U}$ on the optimal MMAP value.

Performing the depth-first lookaheads at every single iteration can often incur significant overhead. To bound this overhead, we use a cutoff parameter $\theta$ to trigger the depth-first lookaheads every $\theta$ best-first iterations (see line 6). It is also important to note that the cache information is shared 


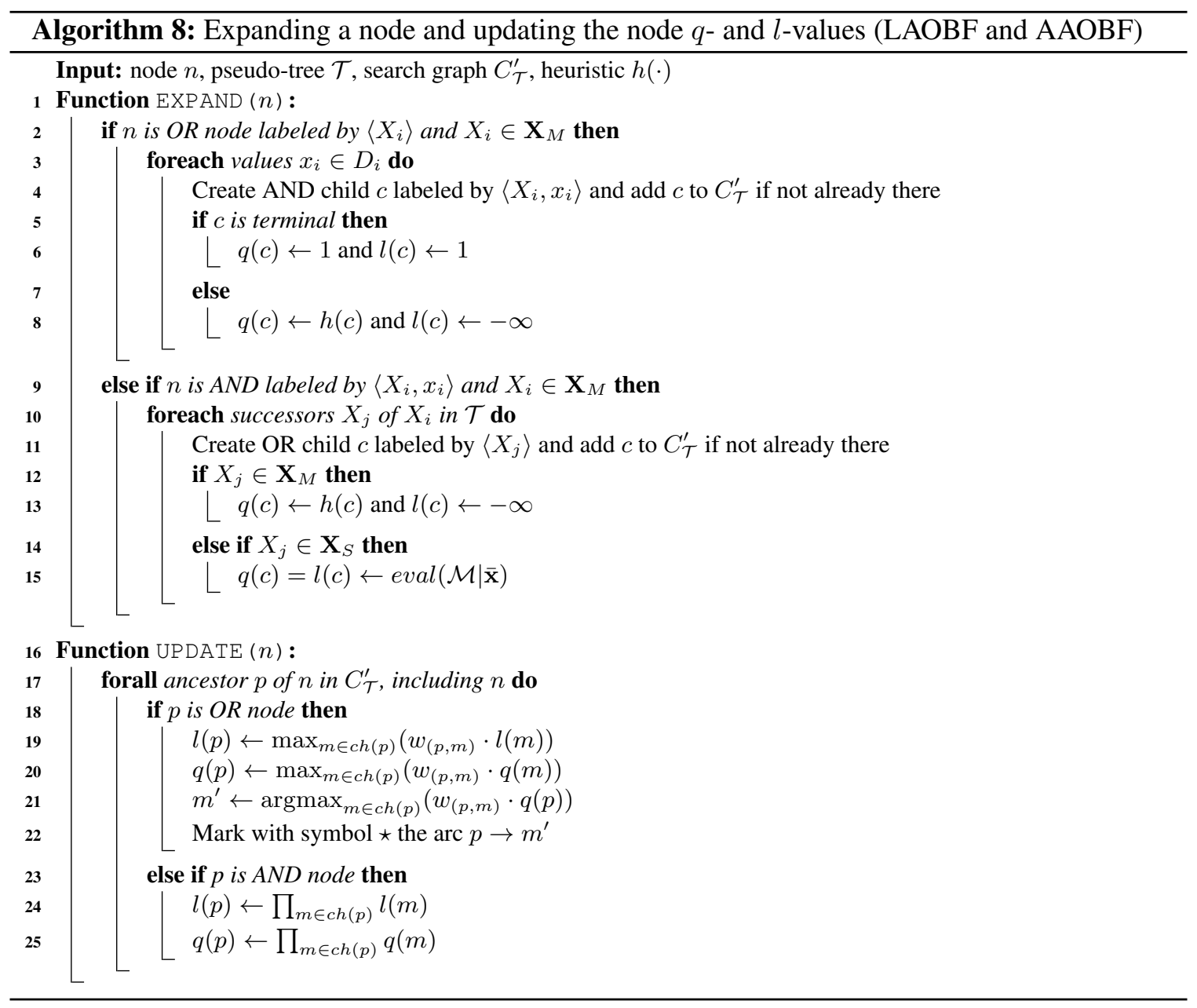

between the depth-first and best-first iterations, so that the solutions to the summation subproblems can be reused.

\subsubsection{AAOBF: Alternating Best-First with Depth-First AND/OR Search}

LAOBF described above restricts the depth-first exploration to the subspaces below the tip nodes of the current best partial solution tree $T_{b}$, and, as we will show in the experimental section, this may not always lead to improved lower bounds. Moreover, we find that LAOBF requires carefully tuning the cutoff parameter, which may be challenging. Therefore, our second approach called Alternating Depth-First and Best-First AND/OR Search (AAOBF) is a parameter-free best+depth hybrid that aims to diversify the depth-first exploration (thus allowing it to explore feasible solutions in a greedier manner, rather than restricting it to specific regions dictated by the best-first search process).

Specifically, AAOBF (Algorithm 10) alternates between a depth-first and a best-first stage and maintains two independent partial solution trees for each. In its depth-first stage it expands, depthfirst, a current feasible partial solution tree $T_{l}$ (lines 4-12), where $T_{l}$ is a partial assignment that can be extended to a feasible (often suboptimal) solution, to improve the global lower bound. In its best-first stage it expands, best-first, a current best partial solution tree $T_{b}$ (lines 13-30) to improve 


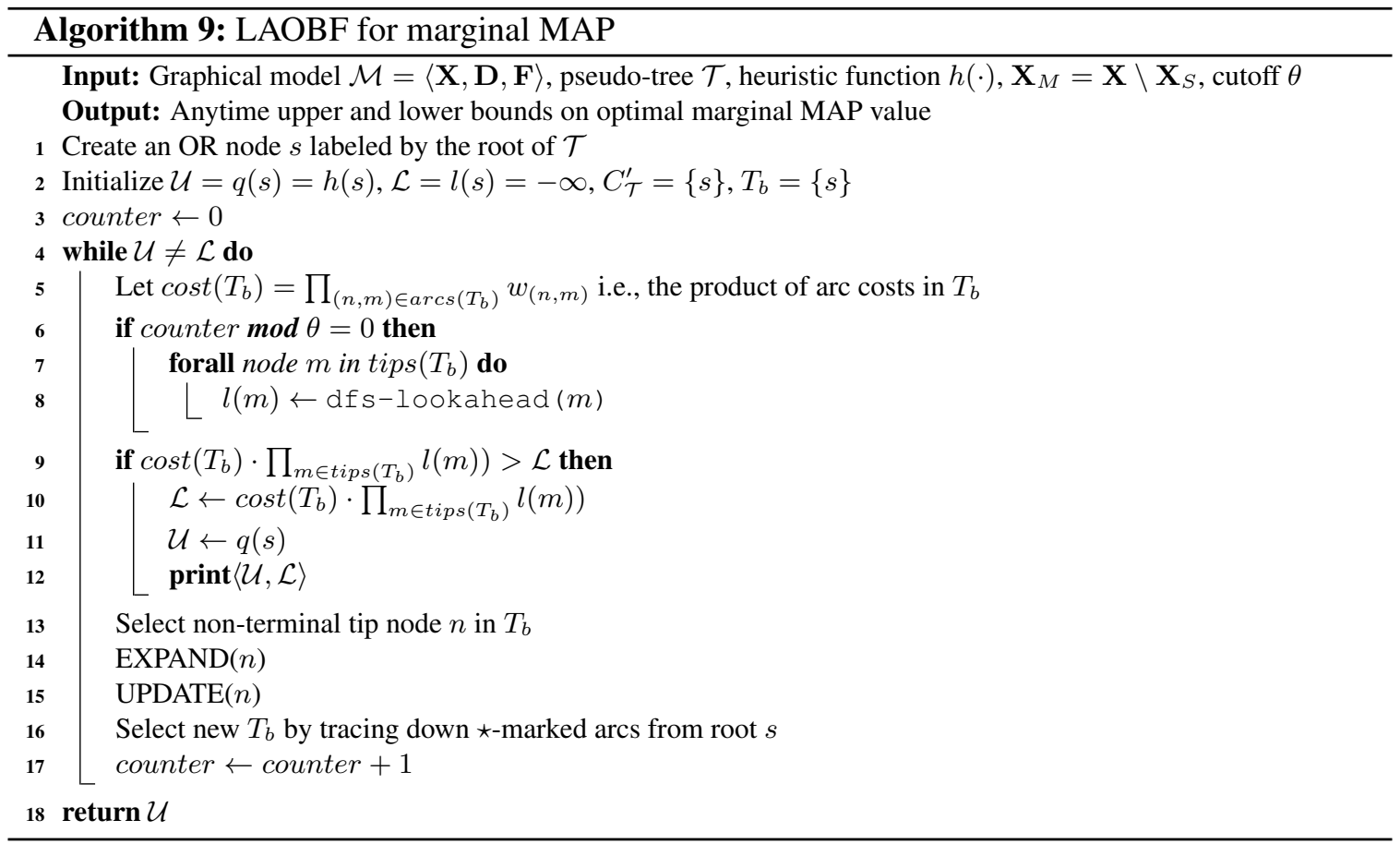

the global solution and an associated upper bound and guide the exploration closer to the region containing the optimal solution. At any stage during search, the partial solution trees $T_{l}$ and $T_{b}$ can be identified by tracing down $\diamond$ - and $\star$-marked arcs (respectively) from the root $s$ of $G_{\mathcal{T}}^{\prime}$.

The algorithm begins by expanding non-terminal tip nodes from the current $T_{l}$. Each node expansion is followed by a bottom-up revision of the $q$ - and $l$-values (lines 6-7). An OR node which was just expanded also marks with a $\diamond$ symbol the arc to its best AND child (lines 8-10). Notice that the $\diamond$-markings do not change during this stage, and therefore $T_{l}$ is extended depth-first to a solution tree.

When a solution is found (i.e., $T_{l}$ has no tips), AAOBF attempts to give control to best-first search. Before turning to best-first search, it revises the $\diamond$-markings along the $\operatorname{arcs}$ in $G_{\mathcal{T}}^{\prime}$ so that a new feasible partial solution tree could be selected later. Specifically, if there is a lower bound $l(n)$ at node $n$ (i.e., $l(n) \neq-\infty$ ) then AAOBF marks with a $\diamond$ symbol the arc to the AND child that minimizes the ratio between the current $l(n)$ and the successor's updated $q$-value, and therefore is the most likely to increase $l(n)$ (line 19). Otherwise, it selects the best AND child having the largest heuristic (upper bounding) value (line 21).

AAOBF continues by expanding nodes from $T_{b}$ in the usual best-first manner. However, if during this stage a new feasible partial solution tree $T_{l}$ is identified (line 29), the algorithm switches immediately to the depth-first expansion of the new $T_{l}$. Search terminates with the optimal marginal MAP value when the global lower and upper bounds are equal.

Since the proposed best+depth-first search algorithms for marginal MAP traverse the contextminimal AND/OR search graph, we have that:

THEOREM 2 (complexity). Algorithms LAOBF and AAOBF are sound and complete (namely, they will find an optimal solution if given enough time and space). Their complexity is time $O\left(n \cdot m \cdot k^{w_{c}^{*}}\right.$ 


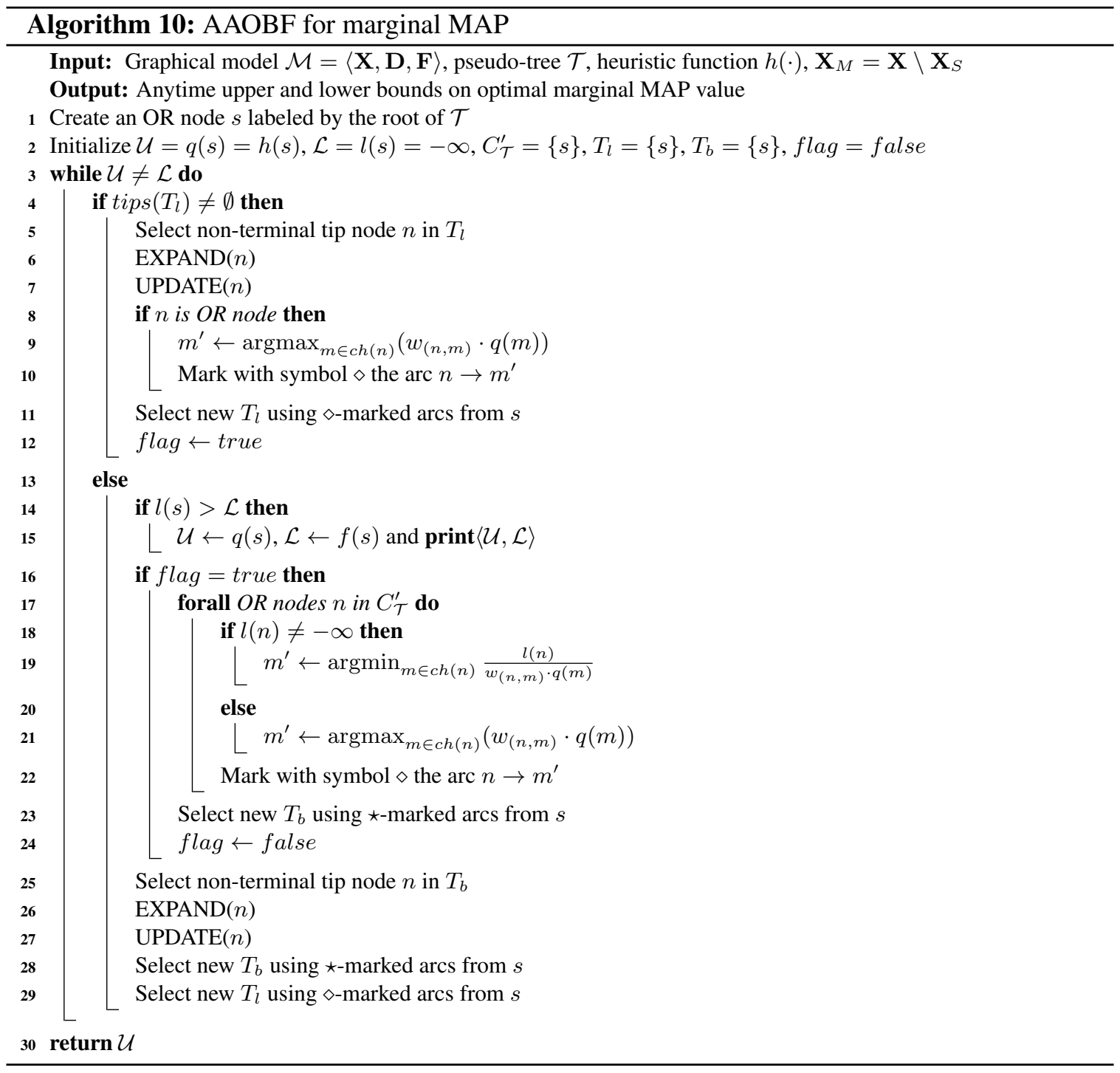

and space $O\left(n \cdot k^{w_{c}^{*}}\right)$, where $n$ is the total number of variables, $m$ is the number of MAP variables $k$ bounds the domain size, and $w_{c}^{*}$ it the induced width of the valid pseudo-tree (i.e., constrained induced width).

Proof. Clearly, the size of the search space explored, and therefore the space complexity of both LAOBF and AAOBF, is bounded by $O\left(n \cdot k^{w_{c}^{*}}\right)$. For both LAOBF and AAOBF, each node expanded during the best-first search stage can be followed in the worst case by a greedy depth-first dive to compute a complete (but not optimal) MAP assignment. Since the depth-first dive takes $O(m)$ time, we have that the time complexity of algorithms LAOBF and AAOBF is $O\left(n \cdot m \cdot k^{w_{c}^{*}}\right)$.

Finally, we note that both LAOBF and AAOBF can switch to using only depth-first tree search as soon as memory fills up, thus continuing to improve the lower bound. 


\subsection{Experimental Results}

In this section, we report results obtained with the anytime search algorithms on the same benchmark domains and using the same evaluation environment as in Section 4.6.

\subsubsection{OVERVIEW AND METHOdOLOGY}

We evaluate algorithms WAOBF, WAOBF-REP, WRBFAOO, LAOBF and AAOBF using the weighted mini-bucket based heuristic function WMB-MM $(i)$. The hyperparameters of the algorithms are set as follows. The initial weight $\alpha_{0}$ used by the weighted best-first AND/OR search algorithms WAOBF, WAOBF-REP and WRBFAOO is 64, while the weight update schedule is $\alpha_{i+1}=\sqrt{\alpha_{i}}$. The cutoff parameter of LAOBF is 1000 .

Since the anytime AND/OR search algorithms generate anytime suboptimal solutions (lower bounds) as well as anytime upper bounds, we developed the anytime performance metrics that focus on the improvement of the quality of anytime solutions and upper bounds over time. In the following subsections, we first show plots depicting the anytime solutions and upper bounds for selected problem instances. Then, we report 3 aggregate metrics to compare the anytime performance of the anytime AND/OR search algorithms, as follows: (1) the number of instances that returned any solution at various time bounds, which compares how fast an algorithm can generate any solution, (2) the average of relative anytime solution qualities as a function of time, which compares the quality of anytime solutions, and (3) the average gap as a function of time, which compares the gap between the upper bound and the anytime solution found by each algorithm. The first metric characterizes how fast an algorithm can generate any solution, regardless of the quality. This fast response time is a desirable property in many real-time applications. The second metric complements the first one by considering the relative solution quality over time, while the last metric characterizes the average quality of anytime solutions by deviations from the corresponding upper bounds.

\subsubsection{RESULTS ON INDIVIDUAL INSTANCES}

We now present the anytime solutions and upper bounds found on selected problem instances from each benchmark. For the weighted best-first AND/OR search algorithms, we compute upper bounds using the weight. Recall that these algorithms guarantee that at termination the generated solution lies within a constant factor $\alpha$ of the optimal solution. In Figure 6, we show plots of the anytime solutions and upper bounds as a function of time for $i$-bounds 12 and 18, respectively. Table 5 reports similar numerical results obtained on additional problem instances.

- On the grid domain, the anytime weighted AND/OR search algorithms perform significantly worse than the hybrid AND/OR search algorithms. For example, in Table 5 we see that on the hard grid 90-34-5 instance, all three weighted AND/OR search algorithms failed to find a single suboptimal solution due to the memory limit, while AAOBF and LAOBF found anytime solutions and upper bounds at the 1 minute time bound and improved them over time. Furthermore, on the hard grid 90-30-5 instance, only WAOBF returned any solution within the 1 minute time bound, but AAOBF and LAOBF found better anytime solutions and upper bounds, respectively. We also observe that both AAOBF and LAOBF generate frequent anytime updates compared to the weighted best-first search algorithms.

- On pedigrees, both algorithms AAOBF and LAOBF found and improved the anytime solutions and upper bounds over time on the hard pedigree 38 , hard pedigree 39 , and hard 

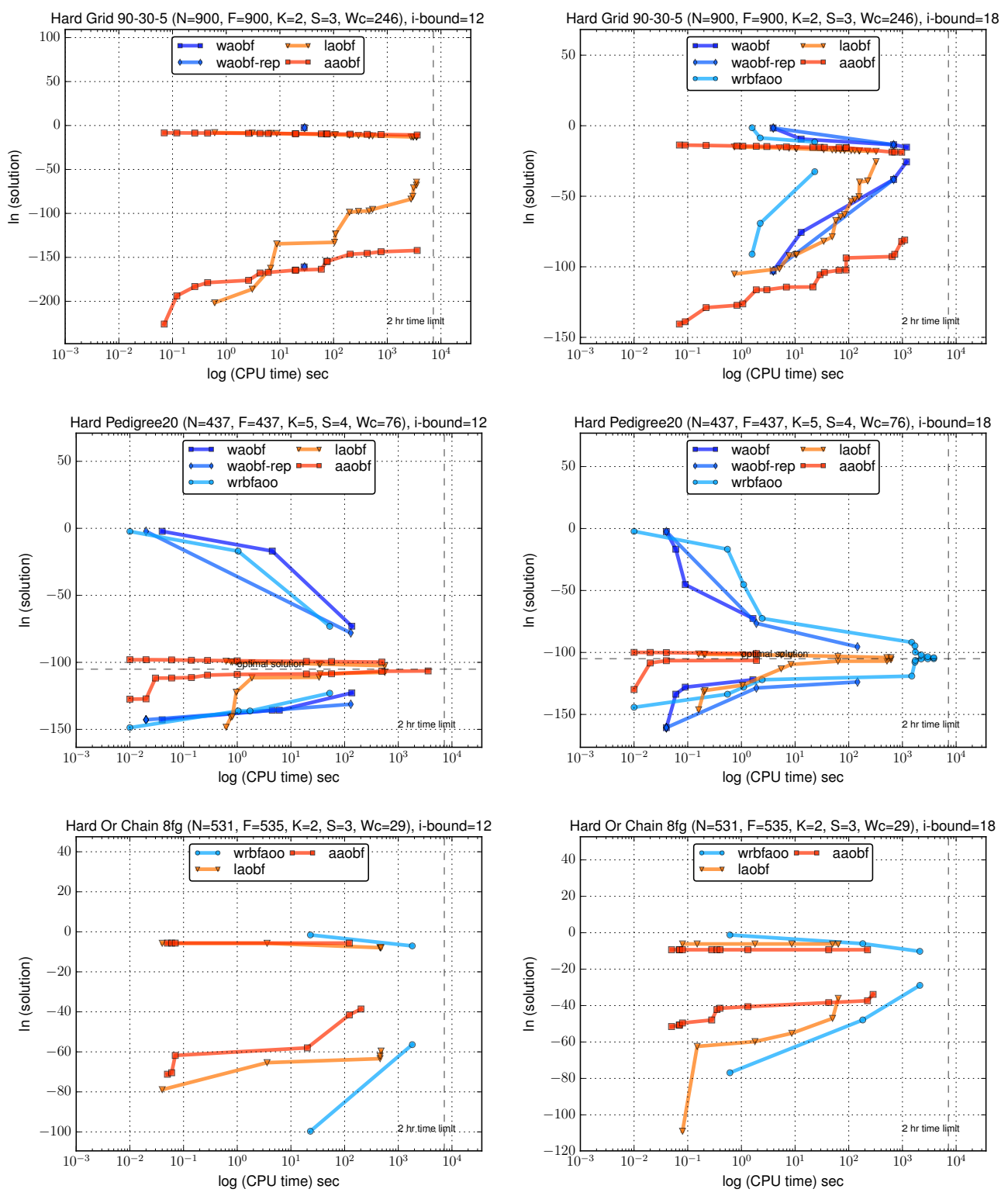

Figure 6: Anytime lower and upper bounds from hard problem instances with $i$-bound 12 (left) and 18 (right). The horizontal axis is the CPU time in log scale and the vertical axis is the value of marginal MAP in log scale. This figure visualizes the improvement of anytime solutions over increased time bounds up to 2 hour, and each data point of the lower curves corresponds to the time that anytime solutions were discovered. The upper curves show the upper bounds. A search algorithm terminates with the optimal solution when upper and lower curves coincided.

pedigree 20 instances. We see WAOBF-REP failed to find a single solution on the hard pedigree 38 and the hard pedigree 39 instances. WAOBF also failed on the hard pedigree 38 
instance due to the memory limit. We can also observe that AAOBF and LAOBF converge faster to the optimal solution on the hard pedigree 20 instance.

- On promedas, algorithms WAOBF, WAOBF-REP, and WRBFAOO failed to find a single solution on the hard or_chain16_fg and the hard or_chain24_fg instances, while both AAOBF and LAOBF found and improved the solutions and upper bounds over time. Moreover, AAOBF and LAOBF produced better anytime solutions than BRAOBB on all reported problem instances with $i$-bounds 12 and 18, respectively.

Overall, from Figure 6 and Table 5, we see that the anytime solutions (lower curves) found by AAOBF and LAOBF are superior to those found by WAOBF, WAOBF-REP, and WRBFAOO, respectively. Furthermore, the distinction of the quality of anytime solution is clear at $i$-bound 12 . Comparing $\mathrm{AAOBF}$ and $\mathrm{LAOBF}$, we can see that $\mathrm{AAOBF}$ found higher quality solutions at earlier time bounds on the hard pedigree 20 and the hard or_chain $8 \_f g$ instances. In addition, the hybrid AND/OR search algorithms produced more frequent anytime solutions compared to the weighted best-first AND/OR search algorithms. WAOBF found only a single anytime solution on the hard grid 90-30-5 instance at $i$-bound 12, while AAOBF and LAOBF found 14 and 15 anytime solution within the same 2-hour time limit, respectively. We also observe that LAOBF and AAOBF produced tighter upper bounds than the weighted best-first search algorithms. Comparing the upper bounds produced by LAOBF and AAOBF, we see that they mostly overlap on the problem instances considered.

\subsubsection{THE NUMBER OF INSTANCES THAT RETURNED ANY SOLUTION AT TIME T}

We next report the number of instances that returned any solution at various time bounds. This metric visualizes how fast each algorithm can produce a solution, regardless of its quality. Figure 7 compares the percentage of problem instances returning feasible solutions at varying time bounds for all competing algorithms at the $i$-bounds 12 and 18, respectively. Note that the dashed lines and the solid lines correspond to the ratio computed from anytime solutions and optimal solutions, respectively.

In Figure 7, we see that RBFAOO solves optimally within time the largest number of instances on all benchmark and at all reported $i$-bounds. However, at the 1 minute time bound and for $i$-bound 18 , the percent of instances exactly solved by RBFAOO is only $61 \%, 42 \%$, and $36 \%$ on the grid, pedigree, and promedas domain, respectively. Considering the fact that the exact best-first AND/OR search algorithms are usually terminated by the memory limit, the anytime AND/OR search algorithms are more desirable for improving the coverage with suboptimal solutions. Comparing the dashed lines, we observe that both AAOBF and LAOBF cover more instances than the weighted best-first AND/OR search algorithms at all reported time bounds.

- On the grid domain with $i$-bound 18, AAOBF and LAOBF covered $98.6 \%$ and $97.3 \%$ of the instances at the 1 minute time bound, while WAOBF, WAOBF-REP, and WRBFAOO covered 93.3\%, 93.3\%, and 92.0\%, respectively. BRAOBB also generated anytime solutions for $97.3 \%$ of the instances. We can see that all anytime AND/OR search algorithms solved more than $30 \%$ of the instances compared to RBFAOO.

- On pedigrees with $i$-bound 18, AAOBF and LAOBF covered $92.0 \%$ and $80.0 \%$ of the instances, respectively, at the 1 minute time bound, while WAOBF, WAOBF-REP, and WRBFAOO covered $80.0 \%, 80.0 \%$, and $84.0 \%$, respectively. BRAOBB covered $54.0 \%$ of the 
(a) grid

\begin{tabular}{|c|c|c|c|c|c|c|c|c|c|}
\hline \multirow{3}{*}{$\begin{array}{c}\text { instance } \\
\left(n, f, k, w_{c}, w_{u}\right)\end{array}$} & \multirow[t]{3}{*}{ algorithm } & \multicolumn{4}{|c|}{$i=12$} & \multicolumn{4}{|c|}{$i=18$} \\
\hline & & \multicolumn{2}{|c|}{$1 \mathrm{~min}$} & \multicolumn{2}{|c|}{$1 \mathrm{hr}$} & \multicolumn{2}{|c|}{$1 \mathrm{~min}$} & \multicolumn{2}{|c|}{$1 \mathrm{hr}$} \\
\hline & & $\mathrm{lb}$ & ub & $\mathrm{lb}$ & ub & $\mathrm{lb}$ & ub & $\mathrm{lb}$ & ub \\
\hline \multirow{6}{*}{$\begin{array}{l}\text { grid hard } \\
90-30-5 \mathrm{I} 1 \\
(900,900,2,246,43)\end{array}$} & AAOBF & -163.581 & -9.54662 & -143.601 & -10.8083 & -103.973 & -15.563 & -80.962 & -18.8718 \\
\hline & LAOBF & -134.606 & -10.0975 & -64.4327 & -13.1226 & $\begin{array}{l}-67.161 \\
\end{array}$ & -17.2402 & -25.4589 & -18.5986 \\
\hline & WAOBF & -160.909 & -2.5142 & - & - & -75.6271 & -9.45339 & -25.663 & -15.2593 \\
\hline & WAOBF-REP & - & - & - & - & - & - & - & - \\
\hline & WRBFAOO & - & - & - & - & - & - & - & - \\
\hline & BRAOBB & -126.653 & - & -126.653 & - & -104.404 & - & -104.404 & - \\
\hline \multirow{6}{*}{$\begin{array}{l}\text { grid hard } \\
90-34-5 \mathrm{I} 1 \\
(1156,1156,2,301,49)\end{array}$} & AAOBF & -235.169 & 1.23546 & -224.245 & 0.937635 & -96.3262 & -11.9388 & -71.4547 & -12.7446 \\
\hline & LAOBF & -194.728 & -0.52338 & -170.317 & -0.85827 & -80.75 & -14.599 & -54.0588 & -15.346 \\
\hline & WAOBF & - & - & - & - & - & - & - & - \\
\hline & WAOBF-REP & - & - & - & - & - & - & - & - \\
\hline & WRBFAOO & - & - & - & - & - & - & - & - \\
\hline & BRAOBB & -253.812 & - & -253.812 & - & -118.629 & - & -106.33 & - \\
\hline
\end{tabular}

(b) pedigree

\begin{tabular}{|c|c|c|c|c|c|c|c|c|c|}
\hline \multirow{3}{*}{$\begin{array}{c}\text { instance } \\
\left(n, f, k, w_{c}, w_{u}\right)\end{array}$} & \multirow[t]{3}{*}{ algorithm } & \multicolumn{4}{|c|}{$i=12$} & \multicolumn{4}{|c|}{$i=18$} \\
\hline & & \multicolumn{2}{|c|}{$1 \mathrm{~min}$} & \multicolumn{2}{|c|}{$1 \mathrm{hr}$} & \multicolumn{2}{|c|}{$1 \mathrm{~min}$} & \multicolumn{2}{|c|}{$1 \mathrm{hr}$} \\
\hline & & $\mathrm{lb}$ & ub & $\mathrm{lb}$ & ub & $\mathrm{lb}$ & ub & $\mathrm{lb}$ & $\mathrm{ub}$ \\
\hline \multirow{6}{*}{$\begin{array}{l}\text { pedigree hard } \\
\text { pedigree38 I1 } \\
(724,724,5,149,62)\end{array}$} & AAOBF & -216.404 & -161.012 & -214.364 & -161.309 & -183.628 & -163.778 & -181.372 & -164.04 \\
\hline & LAOBF & -208.81 & -162.96 & -198.402 & -163.595 & -199.539 & -164.345 & -198.698 & -164.942 \\
\hline & WAOBF & - & - & - & - & - & - & - & - \\
\hline & WAOBF-REP & - & - & - & - & - & - & - & - \\
\hline & WRBFAOO & - & - & -285.035 & -35.6294 & - & - & -184.065 & -109.446 \\
\hline & BRAOBB & -232.987 & - & -212.611 & - & -206.441 & - & -206.441 & - \\
\hline \multirow{6}{*}{$\begin{array}{l}\text { pedigree hard } \\
\text { pedigree39 I1 } \\
(1272,1272,5,132,20)\end{array}$} & AAOBF & -321.692 & -299.275 & -320.595 & -300.202 & -325.879 & -301.936 & -315.208 & -302.987 \\
\hline & LAOBF & -321.044 & -301.09 & -319.8 & -303.019 & -318.817 & -304.423 & -313.72 & -306.28 \\
\hline & WAOBF & -334.015 & -118.092 & -332.306 & -197.591 & -329.458 & -116.481 & -329.458 & -116.481 \\
\hline & WAOBF-REP & - & - & - & - & - & - & - & - \\
\hline & WRBFAOO & -343.759 & -204.401 & -343.759 & -204.401 & -340.981 & -202.749 & -332.914 & -256.712 \\
\hline & BRAOBB & - & - & - & - & - & - & - & - \\
\hline
\end{tabular}

(c) promedas

\begin{tabular}{|c|c|c|c|c|c|c|c|c|c|}
\hline \multirow{3}{*}{$\begin{array}{c}\text { instance } \\
\left(n, f, k, w_{c}, w_{u}\right)\end{array}$} & \multirow[t]{3}{*}{ algorithm } & \multicolumn{4}{|c|}{$i=12$} & \multicolumn{4}{|c|}{$i=18$} \\
\hline & & \multicolumn{2}{|c|}{$1 \mathrm{~min}$} & \multicolumn{2}{|c|}{$1 \mathrm{hr}$} & \multicolumn{2}{|c|}{$1 \mathrm{~min}$} & \multicolumn{2}{|c|}{$1 \mathrm{hr}$} \\
\hline & & $\mathrm{lb}$ & ub & $\mathrm{lb}$ & ub & $\mathrm{lb}$ & ub & $\mathrm{lb}$ & ub \\
\hline \multirow{6}{*}{$\begin{array}{l}\text { promedas hard } \\
\text { or chain } 16 \mathrm{fg} \text { I1 } \\
(1675,1701,2,490,90)\end{array}$} & AAOBF & -226.479 & -18.7189 & -210.327 & -18.7195 & -137.205 & -19.853 & -136.494 & -19.8553 \\
\hline & LAOBF & -192.147 & -18.5613 & -191.184 & -18.5617 & -129.625 & -9.39193 & -120.084 & -9.39332 \\
\hline & WAOBF & - & - & - & - & - & - & - & - \\
\hline & WAOBF-REP & - & - & - & - & - & - & - & - \\
\hline & WRBFAOO & - & - & - & - & - & - & - & - \\
\hline & BRAOBB & -218.756 & - & -218.756 & - & -190.695 & - & -190.695 & - \\
\hline \multirow{6}{*}{$\begin{array}{l}\text { promedas hard } \\
\text { or chain } 24 \mathrm{fg} \mathrm{I} 1 \\
(1155,1172,2,154,71)\end{array}$} & AAOBF & -111.666 & -7.1599 & -88.933 & -7.16056 & -64.8236 & -10.0497 & -64.8236 & -10.0497 \\
\hline & LAOBF & -129.625 & -9.39193 & -120.084 & -9.39332 & -125.953 & -9.79024 & -95.841 & -9.81755 \\
\hline & WAOBF & - & - & - & - & - & - & - & - \\
\hline & WAOBF-REP & - & - & - & - & - & - & - & - \\
\hline & WRBFAOO & - & - & - & - & - & - & - & - \\
\hline & BRAOBB & -166.674 & - & -115.428 & - & -113.823 & - & -113.823 & - \\
\hline
\end{tabular}

Table 5: Lower and upper bounds on the optimal value on 2 selected instances from each benchmark at 1 minute and 1 hour time bound with $i$-bound 12 and 18 . In the table, $l b$ denotes the solution cost at the time bound and $u b$ denotes the upper bound. The highlighted results show best performance for an instance and a time bound. The hybrid schemes seem superior. 

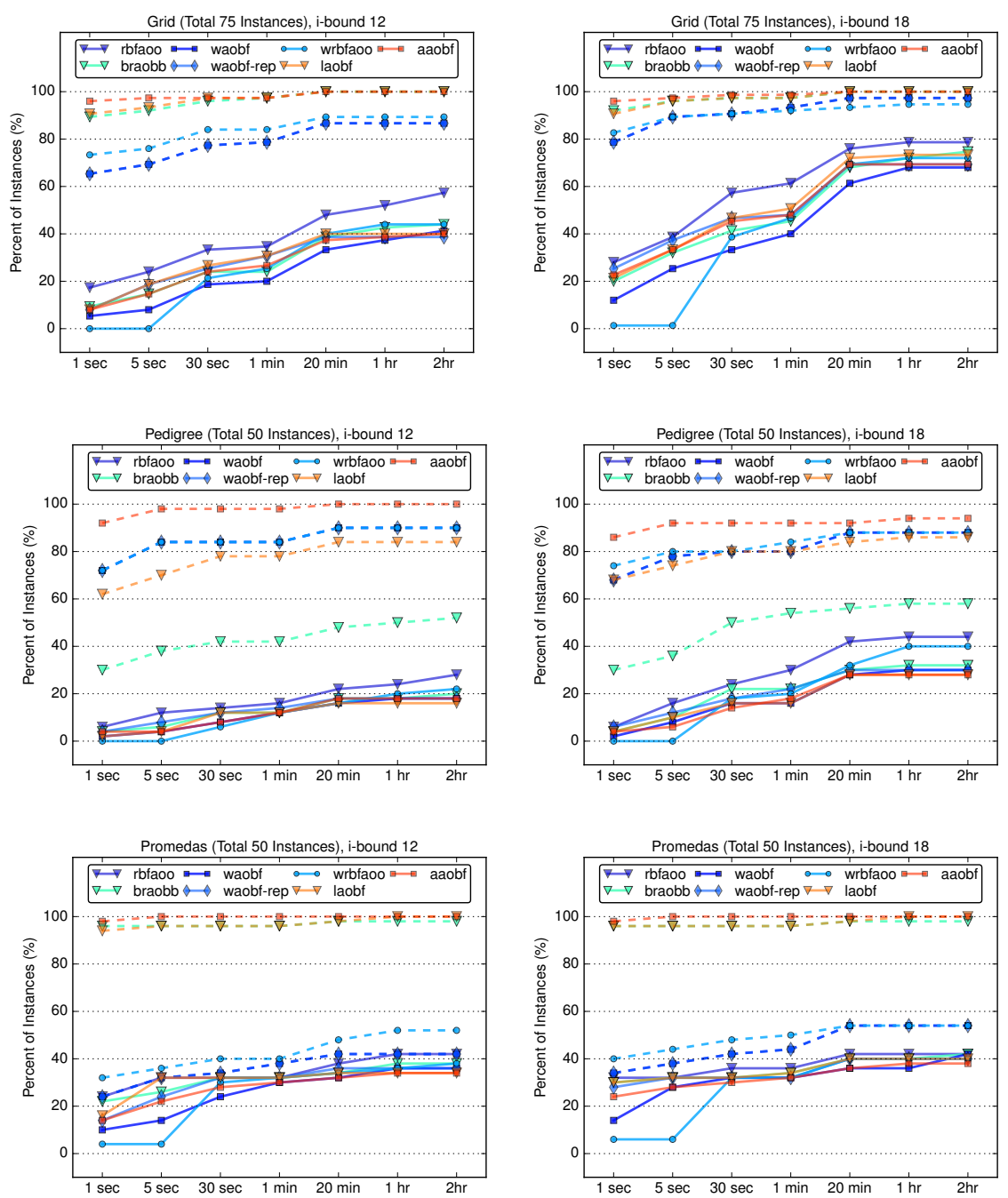

Figure 7: The number of instances returned any solution at time $t$ with the $i$-bound 12 (left) and 18 (right). The horizontal axis is time bounds from 1 second to 2 hours and the vertical axis is the percent of instances solved. The solid lines show the progress of finding optimal solutions with increased time bounds by anytime AND/OR search algorithms. The dashed line corresponds to percent of instances that returned at least one solution within the time bounds. Anytime AND/OR search shows good performance in is ability for finding feasible solutions in a shorter time bounds. In particular, AAOBF and LAOBF returned feasible solutions for more than $90 \%$ of instances on all domains in 5 second time bounds. Overall, AAOBF and LAOBF outperformed other algorithms on this metric.

instances. Compared to RBFAOO, the anytime AND/OR search algorithms covered more than $50 \%$ of the instances. 
- On promedas with $i$-bound 18 , the coverage of AAOBF and LAOBF is $100.0 \%$ and $96.0 \%$ at the 1 minute time bound, while WAOBF, WAOBF-REP, and WRBFAOO covered $44.0 \%$, $44.0 \%$, and $54.0 \%$, respectively. BRAOBB covered $90.0 \%$ of the instances. The trend is quite different in the promedas domain where AAOBF and LAOBF showed more than $60 \%$ improvement over RBFAOO while WAOBF, WAOBF-REP, and WRBFAOO improved over RBFAOO only by $10 \%$.

Overall, we can conclude that AAOBF and LAOBF are the best performing algorithms according to the current metric.

\subsubsection{THE AVERAGE OF RELATIVE ANYTIME SOLUTION QUALITIES AT TIME T}

We next report our second aggregate measure; the average of relative solution qualities as a function of time. We define the relative solution quality of an anytime search algorithm on a measure per instance $i$ at time $t$ by:

$$
\rho_{\text {sol }}^{i}(t)=\frac{\text { The solution cost of algorithm at time } t}{\text { The best solution cost found at time } t \text { by any algorithm }}
$$

Larger values indicate superior solutions (i.e., larger lower bounds). When all anytime search algorithms failed to produce a single anytime solution, we set $\rho_{\text {sol }}^{i}(t)$ to 0 . The average of relative anytime metric is the mean of $\rho_{\text {sol }}^{i}(t)$ over all problem instances a benchmark domain:

$$
\rho_{\text {sol }}(t)=\frac{\sum_{i \in I} \rho_{s o l}^{i}(t)}{|I|}
$$

where $I$ is the set of instances and $|I|$ denotes the total number of instances in a domain.

Figure 8 reports the average relative solution quality obtained on the grid, pedigree and promedas domains, using $i$-bounds 12 and 18, respectively. In this case, RBFAOO can only take two values for $\rho_{\text {sol }}(t)$, namely either 1.0 if it terminated within the time bound or 0.0 otherwise. We observe that all algorithms show superior performance compared with RBFAOO on all benchmark and at almost all reported time bounds.

- On the grid domain with $i$-bound 18 at the 1 minute time bound, the solution qualities of AAOBF, LAOBF, WAOBF, WAOBF-REP, WRBFAOO, and BRAOBB are 0.935, 0.926, 0.887, 0.894, 0.923, and 0.836, respectively. We can see that both AAOBF and LAOBF maintain a higher solution quality compared to the weighted AND/OR search algorithms. In this case BRAOBB performs the worst. The trend is similar for the smaller $i$-bound of 12 .

- On pedigrees with $i$-bound 18 at the 1 minute time bound, the solution qualities of AAOBF, LAOBF, WAOBF, WAOBF-REP, WRBFAOO, and BRAOBB are 0.931, 0.799, 0.802, 0.794, 0.836 , and 0.528 , respectively. Here, AAOBF is still superior while LAOBF and the weighted best-first search algorithms perform similarly to each other. BRAOBB is inferior to AAOBF and the gap remains consistent over time. Again, the trend is similar for $i$-bound 12 .

- On promedas domain with $i$-bound 18 at the 1 minute time bound, the solution qualities of AAOBF, LAOBF, WAOBF, WAOBF-REP, WRBFAOO, and BRAOBB are 0.907, 0.841, 0.414, $0.415,0.460$, and 0.877 , respectively. We can see that AAOBF and LAOBF maintain higher solution quality compared to the weighted AND/OR search algorithms. The improvement of 

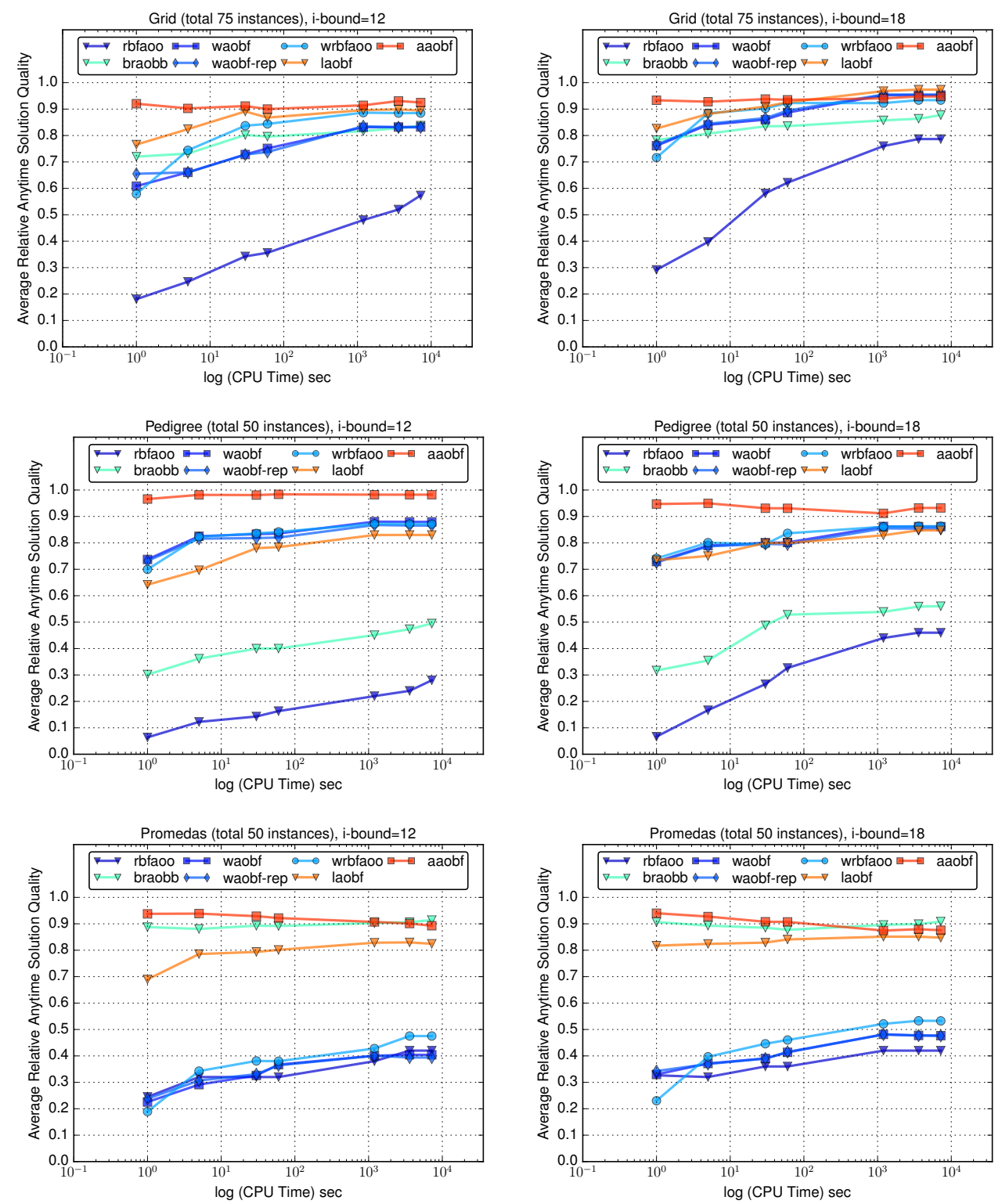

Figure 8: The average relative anytime solution qualities with $i$-bound 12 (left) and 18 (right). The horizontal axis is the time bounds from 1 second to 2 hour and the vertical axis is the relative anytime solution quality ranging from 0 to 1 . Each line shows the changes of the solution quality over varying time bounds for a search algorithm. On the average, the hybrid search algorithms obtain the best solution quality in most cases and the quality measure approaches 1.0.

the solution quality achieved by weighted best-first search compared to RBFAOO is not as significant $(<0.1)$. We also see that BRAOBB is the best performing algorithm at longer time 
bounds. For example, the solution quality at the 2-hour time bound of BRAOBB and AAOBF is 0.909 and 0.876 , respectively.

Overall, we see that AAOBF and LAOBF produced higher quality anytime solutions than the other anytime AND/OR search algorithms across benchmarks and $i$-bounds. The weighted best-first search algorithms have similar relative solution quality but WRBFAOO consistently outperforms WAOBF and WAOBF-REP, respectively.

\subsubsection{THE AVERAGE GAP OF ANYTIME SOLUTIONS AND UPPER BOUNDS AT TIME T}

The last metric considered is the average gap for a benchmark domain at time $t$. We define the gap at time $t$ for algorithm $\mathrm{A}$ as the ratio between the logarithm of the upper bound $\mathcal{U}$ generated by $\mathrm{A}$ and the logarithm of the solution cost $\mathcal{L}$ (lower bound) at time $t$, as follows:

$$
\gamma_{A}(t)= \begin{cases}\frac{\alpha-\beta}{\alpha} & \text { if } 0 \leq \mathcal{L}, \mathcal{U} \leq 1 \\ \frac{\alpha}{\alpha-\beta} & \text { if } 0 \leq \mathcal{L} \leq 1 \text { and } 1 \leq \mathcal{U}\end{cases}
$$

where $\alpha=\log ($ solution cost at $t)$ and $\beta=\log$ (upper bound at $t$ ). For the weighted best-first search algorithms, we set $\beta=\frac{\alpha}{w}$ and always use the first formula in Equation 5. Therefore, $0 \leq \gamma_{A}(t) \leq 1$, and $\gamma_{A}(t)$ is 0 only if an anytime algorithm founds an optimal solution. When an algorithm failed to find a single anytime solution from an individual instance, we set $\gamma_{A}(t)$ to 1.0. The average gap is the mean of $\gamma_{A}(t)$ over all problem instances in the benchmark domain.

Figure 9 shows the average gap between the upper and the lower bounds of the algorithms that are able to provide both upper and lower bounds, using $i$-bounds 12 and 18, respectively. Therefore, algorithms RBFAOO and BRAOBB are excluded.

- On the grid domain with $i$-bound 18 at the 1 minute time bound, the average gap qualities of AAOBF, LAOBF, WAOBF, WAOBF-REP, and WRBFAOO are 0.127, 0.095, 0.144, 0.124, and 0.126 , respectively. We observe that LAOBF is the best and AAOBF follows. The weighted best-first algorithms have similar gap qualities. The trend is the same at longer time bounds.

- On pedigree with $i$-bound 18 at the 1 minute time bound, the average gap qualities of AAOBF, LAOBF, WAOBF, WAOBF-REP, and WRBFAOO are 0.133, 0.208, 0.286, 0.242, and 0.283 , respectively. Here, AAOBF shows notable superiority compared to LAOBF, and the overall trend remains similar to the results from the other benchmarks.

- On promedas with $i$-bound 18 at the 1 minute time bound, the average gap qualities of AAOBF, LAOBF, WAOBF, WAOBF-REP, and WRBFAOO are 0.531, 0.496, 0.607, 0.577, and 0.562 , respectively. Again, AAOBF and LAOBF perform better than the corresponding weighted best-first search algorithms.

In summary, we see that the hybrid AND/OR search algorithms have superior average gap quality compared to the weighted best-first AND/OR search algorithms across all benchmark domains considered.

\section{Related Work}

Solving marginal MAP exactly by depth-first branch and bound search was first introduced in Park and Darwiche (2003) who developed an unconstrained join-tree based upper bound to guide the 

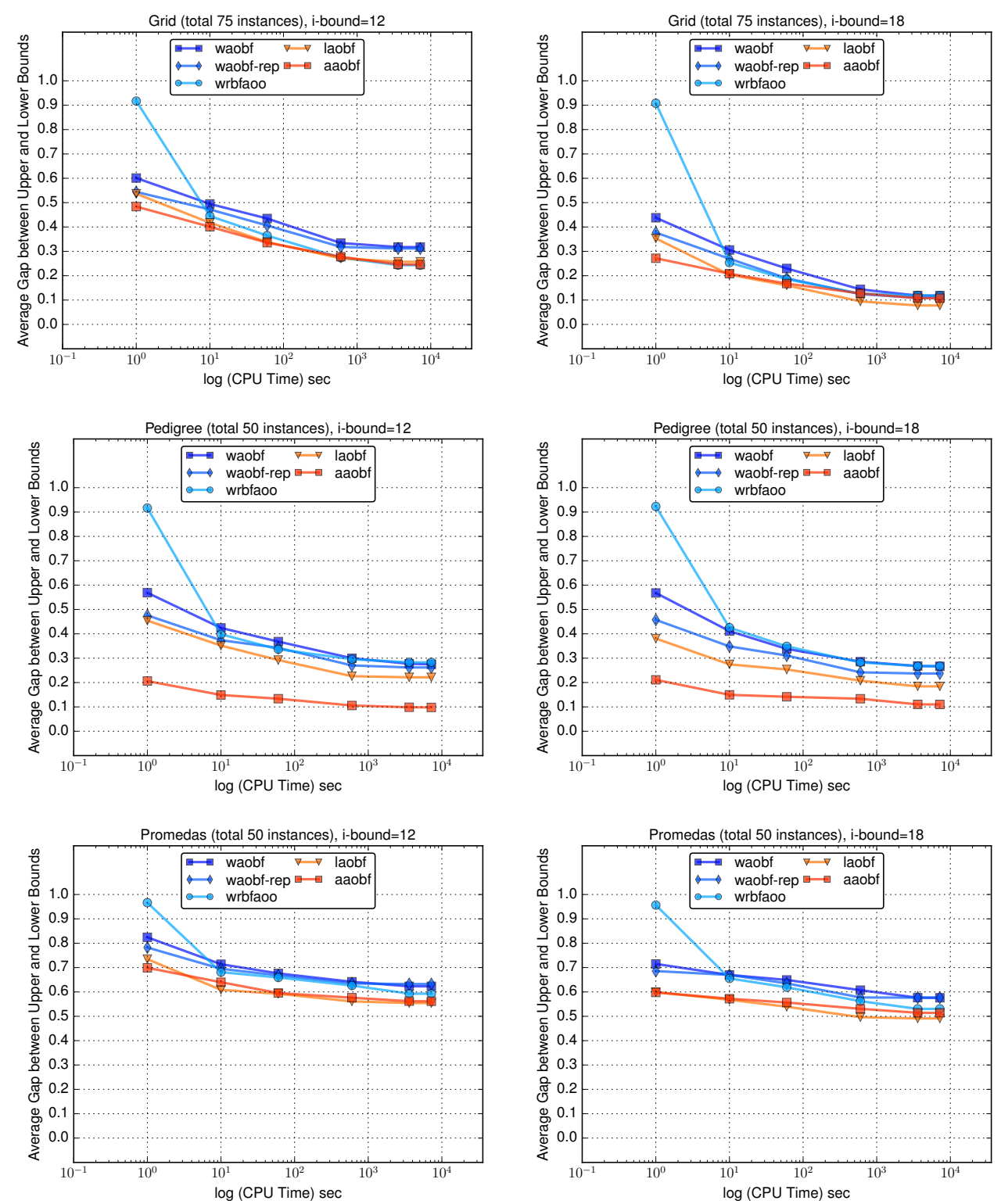

Figure 9: The average relative anytime gap qualities with the $i$-bound 12 (left) and 18 (right). Each line corresponds to a search algorithm that produce both the upper bounds and feasible solutions. The average gap between upper and lower bounds of all search algorithms decrease with time, and AAOBF produced high quality solutions quickly compared with the other algorithms.

search. The join-tree is fully re-valuated at each search node in order to compute upper bounds for all uninstantiated MAP variables simultaneously which allows for dynamic variable orderings. Subsequently, Yuan and Hansen (2009) proposed an incremental evaluation of the unconstrained 
join-tree based upper bound which reduces significantly the computational overhead during search. However, this requires the search to be conducted along a static variable ordering.

Approximation algorithms for marginal MAP, including message passing and variational methods, have also been introduced (Liu \& Ihler, 2013; Jiang, Rai, \& Daume, 2011; Cheng, Chen, Dong, Xu, \& Ihler, 2012; Ping et al., 2015). Upper and lower bound versions of these methods are closely related to the WMB heuristic we use to guide the search. However, unlike our search based algorithms, these schemes do not guarantee eventual optimality of their solutions in an anyspace way, i.e., without significantly increasing memory requirements.

The anytime marginal MAP algorithm introduced recently by Maua and Campos (2012) is another approach that can provide upper and lower bounds. It is an iterative join-tree based algorithm that propagates sets of messages (called factor sets) between the clusters of the join tree. These messages, however, tend to grow very large and therefore the method is limited to solving relatively easy problems with small induced widths.

The exact AND/OR search algorithms for marginal MAP was first introduced by (Marinescu et al., 2014) and the anytime AND/OR search by extending exact best first search by weighted best first search scheme was introduced by (Lee et al., 2016). The hybrid of depth-first and best-first AND/OR search algorithms that provides both upper and lower bounds was introduced by (Marinescu et al., 2017). This paper presents the above AND/OR search algorithms and related algorithms for marginal MAP with additional proofs and extended evaluations from coherent benchmark domains.

Hybrids of depth-first and best-first search The idea of combining depth-first and best-first search was first described in Pearl (1984). A similar idea was also employed in mixed integer programming algorithms as a plunging strategy in Achterberg (2007). The work that is closest in spirit to our LAOBF is that by Stern et al. (2010) who developed an A* with depth-first lookaheads in the context of generic path-finding. More recently, (Allouche, de Givry, Katsileros, Schiex, \& Zytnicki, 2015) introduced a hybrid best-first search algorithm for solving Weighted CSPs that can be guided by a tree decomposition. It selects a node in the best-first manner and performs standard depth-first search with an adaptive number of backtracks to expand the frontier.

\section{Conclusions}

In this paper, we developed depth-first and best-first search algorithms for marginal MAP that explore the compact AND/OR context minimal search space for graphical models, guided by pre-compiled weighted mini-bucket with cost-shifting schemes. We focused on the algorithms as exact solvers at first, and then extended them into anytime algorithms that produce improved solutions with time as well as tightened upper and lower bounds. Through extensive empirical evaluations on a variety of benchmarks we demonstrate the effectiveness of these algorithms as exact schemes against previous unconstrained join-tree based methods, which we also extend to be sensitive to high induced-width models. Our results show not only orders of magnitude improvements over the existing methods, but also the ability to optimally solve many instances that could not be solved before. In particular, the limited memory recursive best-first AND/OR search scheme consistently solved more problems within a two hour time-bound and in many cases was faster, by orders of magnitude, compared with all depth-first OR search counterparts. It emerges as the superior exact scheme overall.

Our proposed new anytime search algorithms for marginal MAP algorithms are based on either weighted search or interleaving best-first and depth-first search. These schemes are able to compute not only anytime lower bounds, but also anytime upper bounds on the optimal marginal MAP value, 
and the gap between the two can be used to better gauge the solution quality during search. Our extensive empirical evaluation on various benchmarks demonstrates the effectiveness of the new algorithms compared with state-of-the-art depth-first search. We are able to show conclusively that our new best+depth-first search approach produces superior quality solutions more quickly than the pure best-first or depth-first search as well as the weighted schemes. They also give tighter gap guarantees compared to weighted best-first search.

The immediate question called for is how to extend our schemes to cases where the conditioned summation task is not tractable (remember that we addressed only cases when the number of MAP variables were large enough to bound the hardness of the summation task). So, for future work we plan to extend our proposed anytime search schemes to also handle effectively marginal MAP problems where the conditioned summation subproblem is not tractable anymore. We also plan to explore parallel search schemes for solving marginal MAP queries. Finally, we will extend to marginal MAP recent look-ahead and subproblem ordering ideas that proved effective for pure optimization tasks (Lam, Kask, Larrosa, \& Dechter, 2017, 2018).

\section{References}

Achterberg, T. (2007). Constraint Integer Programming. Ph.D. thesis, Zuse Institute Berlin.

Allouche, D., de Givry, S., Katsileros, G., Schiex, T., \& Zytnicki, M. (2015). Anytime hybrid best-first search with tree decomposition for weighted csp. In International Conference on Principles and Practice of Constraint Programming, pp. 12-29.

Chakrabarti, P., Ghose, S., \& Sarkar, S. D. (1987). Admissibility of AO* when heuristics overestimate. Artificial Intelligence, 34(1), 97-113.

Cheng, Q., Chen, F., Dong, J., Xu, W., \& Ihler, A. (2012). Approximating the sum operation for marginal-map inference. In Proceedings of the Twenty-Sixth AAAI Conference on Artificial Intelligence, AAAI'12, pp. 1882-1887. AAAI Press.

Cooper, G. (1990). The computational complexity of probabistic inferences. Artificial Intelligence, 42, 393-405.

de Kleer, J., Mackworth, A. K., \& Reiter, R. (1990). Characterizing diagnoses. In Proceedings of the 8th National Conference on Artificial Intelligence. Boston, Massachusetts, July 29 - August 3, 1990, 2 Volumes., pp. 324-330.

Dechter, R. (1999). Bucket elimination: A unifying framework for reasoning. Artificial Intelligence, 113(1-2), 41-85.

Dechter, R., Kask, K., \& Larrosa, J. (2001). A general scheme for multiple lower bound computation in constraint optimization. In Principles and Practice of Constraint Programming, pp. 346360.

Dechter, R., \& Mateescu, R. (2007). AND/OR search spaces for graphical models. Artificial Intelligence, 171(2-3), 73-106.

Dechter, R., \& Pearl, J. (1985). Generalized best-first search strategies and the optimality of A*.. In Journal of ACM, 32(3), 505-536.

Dechter, R., \& Rish, I. (2003). Mini-buckets: A general scheme of approximating inference. Journal of $A C M, 50(2), 107-153$. 
Dechter, R. (2013). Reasoning with Probabilistic and Deterministic Graphical Models: Exact Algorithms. Synthesis Lectures on Artificial Intelligence and Machine Learning. Morgan \& Claypool Publishers.

Dechter, R., \& Mateescu, R. (2007). AND/OR search spaces for graphical models. Artificial Intelligence, 171(2-3), 73-106.

Elidan, G., Globerson, A., \& Heinemann, U. (2012). PASCAL 2011 probabilistic inference challenge. http://www.cs.huji.ac.il/project/PASCAL/.

Flerova, N., Marinescu, R., \& Dechter, R. (2017). Weighted heuristic anytime search: new schemes for optimization over graphical models. Ann. Math. Artif. Intell., 79(1-3), 77-128.

Freuder, E. C., \& Quinn, M. J. (1985). Taking advantage of stable sets of variables in constraint satisfaction problems. In Proceedings of the 9th International Joint Conference on Artificial Intelligence. Los Angeles, CA, USA, August 1985, pp. 1076-1078.

Geffner, H., \& Bonet, B. (2013). A Concise Introduction to Models and Methods for Automated Planning. Synthesis Lectures on Artificial Intelligence and Machine Learning. Morgan \& Claypool Publishers.

Globerson, A., \& Jaakkola, T. (2007). Approximate inference using conditional entropy decompositions. In International Conference on Artificial Intelligence and Statistics (AISTATS), pp. $130-138$.

Ihler, A., Flerova, N., Dechter, R., \& Otten, L. (2012). Join-graph based cost-shifting schemes. In Uncertainty in Artificial Intelligence (UAI), pp. 397-406.

Jiang, J., Rai, P., \& Daume, H. (2011). Message-passing for approximate map inference with latent variables. In Shawe-Taylor, J., Zemel, R. S., Bartlett, P. L., Pereira, F., \& Weinberger, K. Q. (Eds.), Advances in Neural Information Processing Systems 24, pp. 1197-1205. Curran Associates, Inc.

Kishimoto, A., \& Marinescu, R. (2014). Recursive best-first AND/OR search for optimization in graphical models. In Uncertainty in Artificial Intelligence (UAI), pp. 400-409.

Korf, R. (1993). Linear-space best-first search. Artificial Intelligence, 62(1), 41-78.

Lam, W., Kask, K., Larrosa, J., \& Dechter, R. (2017). Residual-guided look-ahead in AND/OR search for graphical models. J. Artif. Intell. Res., 60, 287-346.

Lam, W., Kask, K., Larrosa, J., \& Dechter, R. (2018). Subproblem ordering heuristics for AND/OR best-first search. J. Comput. Syst. Sci., 94, 41-62.

Lee, J., Marinescu, R., Dechter, R., \& Ihler, A. (2016). From exact to anytime solutions for marginal MAP. In 30th AAAI Conference on Artificial Intelligence, pp. 1749-1755.

Lee, J., Marinescu, R., \& Dechter, R. (2014). Applying marginal map search to probabilistic conformant planning: Initial results.. In AAAI Workshop: Statistical Relational Artificial Intelligence.

Liu, Q., \& Ihler, A. (2011). Bounding the partition function using Hölder's inequality. In International Conference on Machine Learning (ICML), pp. 849-856.

Liu, Q., \& Ihler, A. (2013). Variational algorithms for marginal MAP. Journal of Machine Learning Research, 14, 3165-3200. 
Marinescu, R., \& Dechter, R. (2009a). AND/OR branch-and-bound search for combinatorial optimization in graphical models. Artificial Intelligence, 173(16-17), 1457-1491.

Marinescu, R., \& Dechter, R. (2009b). Memory intensive AND/OR search for combinatorial optimization in graphical models. Artificial Intelligence, 173(16-17), 1492-1524.

Marinescu, R., Dechter, R., \& Ihler, A. (2014). AND/OR search for marginal MAP. In Uncertainty in Artificial Intelligence (UAI), pp. 563-572.

Marinescu, R., Dechter, R., \& Ihler, A. (2015). Pushing forward marginal MAP with best-first search. In International Joint Conference on Artificial Intelligence (IJCAI), pp. 696-702.

Marinescu, R., Kask, K., \& Dechter, R. (2003). Systematic vs non-systematic algorithms for solving the MPE task.. In Uncertainty in Artificial Intelligence (UAI), pp. 394-402.

Marinescu, R., Lee, J., Dechter, R., \& Ihler, A. (2017). Anytime best+depth-first search for bounding marginal MAP. In 31th AAAI Conference on Artificial Intelligence, pp. 1749-1755.

Mateescu, R., \& Dechter, R. (2007). A comparison of time-space scheme for graphical models. In Proceedings of the Twentieth International Joint Conference on Artificial Intelligence, pp. 2346-2352.

Maua, D., \& Campos, C. D. (2012). Anytime marginal MAP inference. In International Conference on Machine Learning, pp. 1471-1478.

Mauá, D. D. (2016). Equivalences between maximum a posteriori inference in bayesian networks and maximum expected utility computation in influence diagrams. Int. J. Approx. Reasoning, 68(C), 211-229.

Nilsson, N. J. (1980). Principles of Artificial Intelligence. Tioga, Palo Alto, CA.

Otten, L., \& Dechter, R. (2011). Anytime AND/OR depth-first search for combinatorial optimization. In International Symposium on Combinatorial Search, pp. 117-702.

Park, J., \& Darwiche, A. (2003). Solving MAP exactly using systematic search. In Uncertainty in Artificial Intelligence (UAI), pp. 459-468.

Park, J., \& Darwiche, A. (2004). Complexity results and approximation strategies for MAP explanations. Journal of Artificial Intelligence Research, 21(1), 101-133.

Pearl, J. (1984). Heuristics: Intelligent Search Strategies. Addison-Wesley.

Pearl, J. (1988). Probabilistic Reasoning in Intelligent Systems. Morgan Kaufmann.

Ping, W., Liu, Q., \& Ihler, A. T. (2015). Decomposition bounds for marginal MAP. In Advances in Neural Information Processing Systems 28, pp. 3267-3275.

Pohl, I. (1970). Heuristic search viewed as path finding in a graph. Artificial Intelligence, 1(3-4), 193-204.

Shimony, S., \& Charniak, E. (1991). A new algorithm for finding MAP assignments to belief networks. In P. Bonissone, M. Henrion, L. Kanal, and J. Lemmer (Eds.), Uncertainty in Artificial Intelligence, Vol. 6, pp. 185-193.

Stern, R., Kulberis, T., Felner, A., \& Holte, R. (2010). Using lookahead with optimal best-first search. In 24th AAAI Conference on Artificial Intelligence, pp. 185-190. 
Wainwright, M., Jaakkola, T., \& Willsky, A. (2005). A new class of upper bounds on the log partition function. IEEE Trans. Info. Theory, 51(7), 2313-2335.

Weiss, Y., Yanover, C., \& Meltzer, T. (2007). MAP estimation, linear programming and belief propagation with convex free energies. In Uncertainty in Artificial Intelligence (UAI), pp. 416-425.

Yuan, C., \& Hansen, E. (2009). Efficient computation of jointree bounds for systematic MAP search. In International Joint Conference on Artificial Intelligence (IJCAI), pp. 1982-1989. 